\title{
Phase diagram of the Heisenberg spin ladder with ring exchange
}

\author{
V. Gritsev, B. Normand, and D. Baeriswyl \\ Département de Physique, Université de Fribourg, Pérolles, CH-1700 Fribourg, Switzerland \\ (Received 24 October 2003; revised manuscript received 23 January 2004; published 25 March 2004)
}

\begin{abstract}
We investigate the phase diagram of a generalized spin-1/2 quantum antiferromagnet on a ladder with rung, leg, diagonal, and ring-exchange interactions. We consider the exactly soluble models associated with the problem, obtain the exact ground states which exist for certain parameter regimes, and apply a variety of perturbative techniques in the regime of strong ring-exchange coupling. By combining these approaches with considerations related to the discrete $Z_{4}$ symmetry of the model, we present the complete phase diagram.
\end{abstract}

DOI: 10.1103/PhysRevB.69.094431

PACS number(s): 75.10.Jm, 71.10.Hf

\section{INTRODUCTION}

A number of experiments conducted in recent years suggest that multiple spin-exchange interactions have a significant role in the quantitative description of the physics of low-dimensional cuprate compounds, and a qualitative one in determining the properties of ${ }^{3} \mathrm{He}$ adsorbed on graphite surfaces. Inelastic neutron-scattering measurements of the spin-wave spectrum in the two-dimensional (2D) cuprate system $\mathrm{La}_{2} \mathrm{CuO}_{4}$ (Ref. 1) and in the quasi-1D spin-ladder compound $\mathrm{La}_{6} \mathrm{Ca}_{8} \mathrm{Cu}_{24} \mathrm{O}_{41}$, ${ }^{2}$ as well as two-magnon Ramanscattering measurements on doped systems related to the latter $^{3}$ indicate the presence of contributions from a four-spin cyclic exchange interaction on the order of $10-20 \%$ of the nearest-neighbor superexchange. Measurements of the magnetization and heat capacity of ${ }^{3} \mathrm{He}$ films of various fillings adsorbed on graphite surfaces ${ }^{4,5}$ have been interpreted ${ }^{6}$ in terms of cyclic three-, four- and higher-spin-exchange processes.

The four-spin-exchange term upon which we will focus here arises at fourth order in a strong-coupling (small $t / U$ ) perturbative expansion of the one-band ${ }^{7}$ and three-band ${ }^{8}$ Hubbard model in 2D, and has been shown in this limit to give the leading correction to the nearest-neighbor Heisenberg model. Recent investigations of the influence of this interaction on physical properties have employed perturbative approaches, ${ }^{9,10}$ spin-wave analysis, ${ }^{11,12}$ numerical exact diagonalization of small clusters, ${ }^{6}$ exact diagonalization in combination with conformal field theory (CFT), ${ }^{13}$ and the density-matrix renormalization-group (DMRG) technique. ${ }^{14-17}$ The majority of these studies is restricted to the regime of weak ring-exchange coupling and to systems with only a nearest-neighbor Heisenberg superexchange interaction in addition to this term.

Here we consider a generalized model to gain further insight into the nature of the phases and phase transitions within this class of system. We investigate a spin ladder which includes antiferromagnetic Heisenberg leg, rung, and diagonal, or cross-plaquette next-neighbor, interactions, as well as a ring-exchange term. This system, depicted schematically in Fig. 1, represents the minimal model possessing both the possibility of a four-spin cyclic exchange interaction and, by virtue of the diagonal coupling, points in parameter space with nontrivial exact solutions.
The ring-exchange interaction may be considered to be composed of different four-spin coupling terms between the spins of a plaquette, subject to the special condition that the effective leg-leg coupling is equal to the rung-rung interaction, and equal in magnitude but opposite in sign to the diagonal-diagonal coupling. Relaxing this constraint on the possible four-spin terms results in a more general parameter space with still richer phase behavior, but we are unaware of studies of this model for arbitrary values of all coupling parameters. In addition to the ring-exchange term, a further possible section of this general parameter space is given by the composite-spin representation of a $S=1$ bilinearbiquadratic chain, ${ }^{18}$ which corresponds to the choice of equal leg-leg and diagonal-diagonal couplings. For this specific choice there is an explicit mapping between the spin-1/2 ladder model and the spin-1 chain. In general, the different phases of the spin model, and the transitions between these, may be distinguished by considering the expectation values of nonlocal quantities, such as the string order parameter first introduced $^{19}$ in studies of the spin-1 chain and the LiebSchulz-Mattis twist operator. ${ }^{20}$ The differing effects of the four-spin coupling terms on string order parameters defined for the spin-1/2 ladder were analyzed in Ref. 21.

We begin by considering the points within the parameter space for which exactly soluble models exist, and then analyze those regimes in the vicinity of these points which are accessible by perturbative techniques based on the CFT corresponding to each exact solution, i.e., to the regions where the soluble models are critical. The ladder Hamiltonian which we study and its associated soluble models are based on a plaquette algebra, by which is meant an algebra of op-

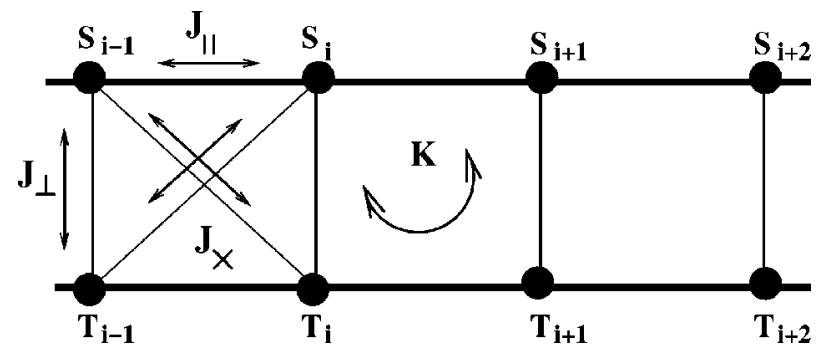

FIG. 1. Generalized spin-ladder system with rung, leg, and diagonal superexchange interactions, $J_{\perp}, J_{\|}$, and $J_{\times}$, and four-spin ring-exchange interaction $K$. Each site contains a spin $S=1 / 2$. 


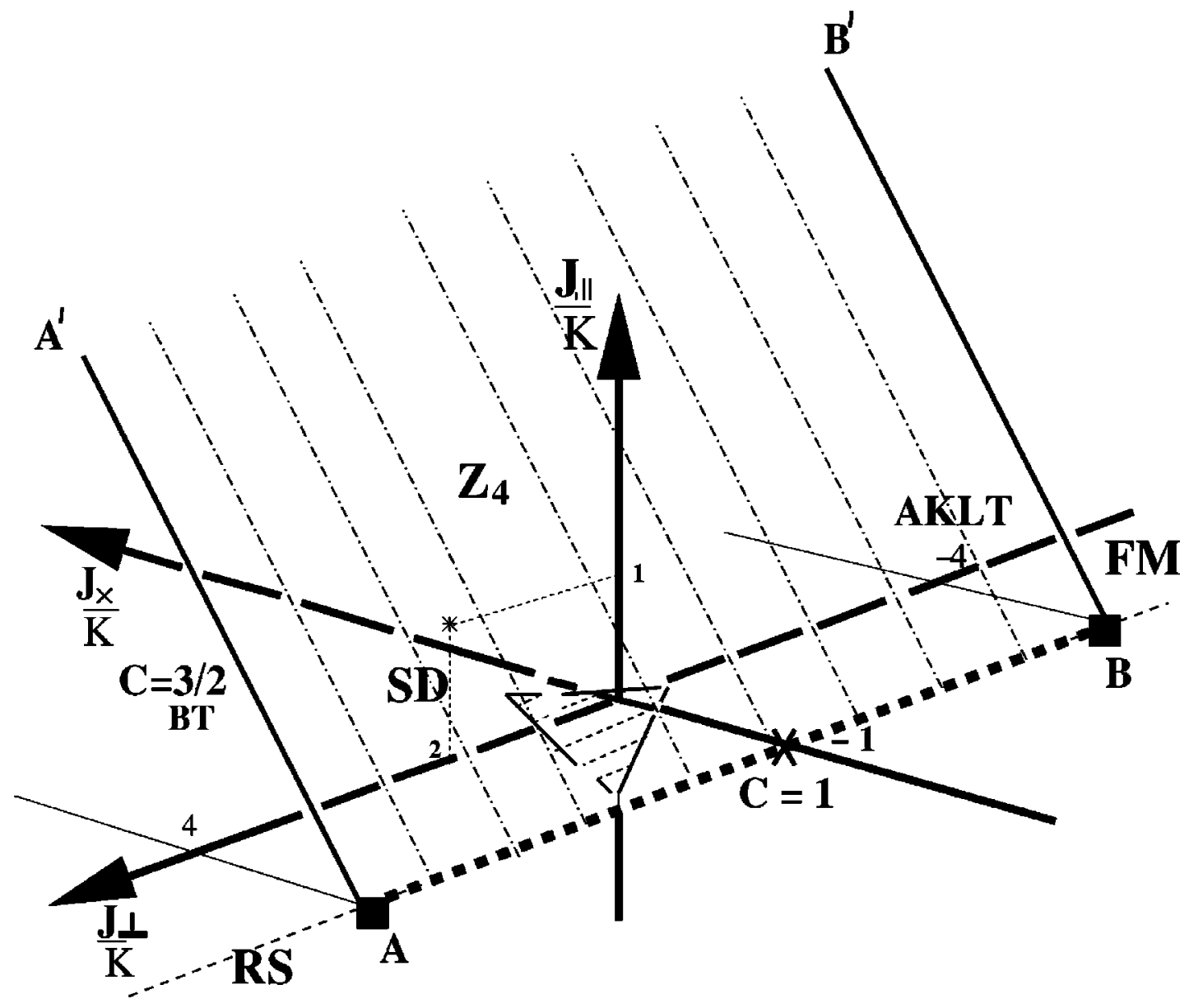

FIG. 2. Phase diagram of the generalized ladder model of Eq. (1), represented as a function of the coupling ratios $J_{\perp} / K, J_{\|} / K$, and $J_{\times} / K$. The inclined plane indicated by dot-dashed lines is invariant with respect to a $Z_{4}$ symmetry, and is given by the equation $J_{\|}-J_{\times}$ $=K$. The dashed line passing through $\mathrm{A}$ and $\mathrm{B}$ represents an exactly soluble model with central charge $c=1$ between $\mathrm{A}$ and $\mathrm{B}$, a "rung-singlet" (RS) phase to the left of point A, and a ferromagnetic (FM) phase to the right of point B. The full line AA' corresponds to a $c=3 / 2$ conformal field theory and belongs to the Babujian-Takhtajan (BT) universality class; it is the exact transition line between the rung-singlet phase and a staggered dimer (SD) phase. The full line $\mathrm{BB}^{\prime}$ separates the ferromagnetic phase from a spin-gapped region of the type proposed by Affleck, Kennedy, Lieb, and Tasaki (AKLT). The shaded triangle lying below the $Z_{4}$-symmetric plane represents a critical surface which is also of the $c=3 / 2 \mathrm{BT}$ universality class.

erators defined on the four-spin plaquette units of the ladder. This algebra satisfies quasilocal commutation relations, meaning that operators defined on next- (and further-) neighbor plaquettes commute. Using the commutation relations of this algebra we have found exact solutions to the YangBaxter equation. $^{22}$ These solutions provide soluble, SU(2)invariant, isotropic spin-ladder Hamiltonians, whose excitation spectrum may be either gapless or gapped. ${ }^{22}$

Gapless, or critical, solutions arise at points or lines of phase transitions, and may be further mapped in the continuum limit to CFTs with a central charge $c \geqslant 1$, or more specifically to Wess-Zumino-Witten (WZW) models. ${ }^{23}$ Perturbative approaches constructed around such WZW models enable one to access regions of parameter space away from these second-order phase-transition points. According to the Zamolodchikov $c$ theorem, ${ }^{24}$ the addition of perturbative conformally noninvariant terms represented by relevant and marginal operators results in a flow either to another CFT with smaller central charge $c$ or to a massive phase. A renormalization-group ( $\mathrm{RG}$ ) procedure constructed on this basis of these perturbations may then be used to establish which fixed points are stable, and to determine the connectivity, the flow structure, and thus the physical properties of different regimes of the phase diagram.

An exact description of the gapped phases is possible by using the idea of "words" on the plaquette algebra to construct variational ground states. The direct relation of this concept to the matrix-product ansatz ${ }^{25}$ allows one to obtain both the exact ground state in suitable parameter regimes and the lowest excited states, from which the lines of phase transitions may also be deduced. For parameter regimes in the vicinity of these exact, gapped solutions, the absence of phase transitions means that many qualitative properties of the associated, gapped phases are known.

Throughout this study we emphasize the role of different symmetries, in particular, the $Z_{4}$ symmetry of the model, in obtaining a complete understanding of the phase diagram. The relevance of this symmetry to the ring-exchange prob- 
lem was first recognized in Ref. 15 in the more restricted context of a "duality" transformation about a self-dual point in the parameter space of the nearest-neighbor ladder model. Here we use the full transformation to relate different soluble models and to develop a perturbative bosonization scheme applicable in the regime of strong ring-exchange coupling.

All of these considerations allow us to deduce the complete phase diagram for the system in the 3D space of coupling ratios $J_{\perp} / K, J_{\|} / K$, and $J_{\times} / K$, which for the orientation of the reader is presented in Fig. 2. The symbols and lines summarized in the caption of Fig. 2, and the phases and transitions they represent, are explained in the course of the analyses to follow. Figure 2 contains as a subset [in the line $\left.\left(J_{\perp} / K=J_{\|} / K, J_{\times}=0\right)\right]$ the $K>0$ part of the phase diagram obtained in the DMRG studies of Läuchli et al., ${ }^{16}$ and confirms the presence of all of the phases and phase transitions proposed in that work. Placing this subset in the context of the full phase diagram permits a significant expansion of our understanding of the nature of these phases, and of the firstand second-order transitions separating them. Further, it provides certain novel, additional phases and transitions inaccessible from the restricted parameter space, yielding additional insight into the competition of superexchange and ring-exchange interactions.

In Sec. II we introduce the Hamiltonian of the model and summarize briefly its associated exact solutions, all of which are critical and belong to different universality classes. Further exact and variational ground states obtained in different regions of parameter space using adapted matrix-product wave functions are presented in Sec. III, accompanied by a discussion of the nature of the associated gapped states and their behavior at phase boundaries. Section IV contains a complete perturbative analysis around the four available CFT solutions, with emphasis on the properties of the strongcoupling (large- $K$ ) regime, which establishes the fixed-point structure of the phase diagram. Section V presents a summary and discussion of the results.

\section{LADDER MODELS}

\section{A. Ring exchange}

The Hamiltonian of the two-leg ladder (Fig. 1) is

$$
\begin{aligned}
H= & J_{\|} \sum_{i}\left(\mathbf{S}_{i} \cdot \mathbf{S}_{i+1}+\mathbf{T}_{i} \cdot \mathbf{T}_{i+1}\right)+J_{\times} \sum_{i}\left(\mathbf{S}_{i} \cdot \mathbf{T}_{i+1}+\mathbf{T}_{i} \cdot \mathbf{S}_{i+1}\right) \\
& +J_{\perp} \sum_{i} \mathbf{S}_{i} \cdot \mathbf{T}_{i}+\frac{1}{2} K \sum_{\square}\left(P_{4}+P_{4}^{-1}\right)
\end{aligned}
$$

where in the last ("ring-exchange") term the four-site permutation operator $P_{4}$ exchanges spins in a cyclic manner around each elementary plaquette $\square$ of the ladder, and is given by the equation

$$
\begin{aligned}
P_{4}+P_{4}^{-1}= & \mathbf{S}_{i} \cdot \mathbf{S}_{i+1}+\mathbf{T}_{i} \cdot \mathbf{T}_{i+1}+\mathbf{S}_{i} \cdot \mathbf{T}_{i}+\mathbf{S}_{i+1} \cdot \mathbf{T}_{i+1} \\
& +\mathbf{S}_{i} \cdot \mathbf{T}_{i+1}+\mathbf{T}_{i} \cdot \mathbf{S}_{i+1}+4\left[\left(\mathbf{S}_{i} \cdot \mathbf{S}_{i+1}\right)\left(\mathbf{T}_{i} \cdot \mathbf{T}_{i+1}\right)\right. \\
& +\left(\mathbf{S}_{i} \cdot \mathbf{T}_{i}\right)\left(\mathbf{S}_{i+1} \cdot \mathbf{T}_{i+1}\right)
\end{aligned}
$$

$$
\left.-\left(\mathbf{S}_{i} \cdot \mathbf{T}_{i+1}\right)\left(\mathbf{S}_{i+1} \cdot \mathbf{T}_{i}\right)\right]+\frac{1}{4} .
$$

We introduce the orthonormal basis

$$
\begin{aligned}
& |0\rangle=\frac{1}{\sqrt{2}}(|\uparrow \downarrow\rangle-|\downarrow \uparrow\rangle), \quad|1\rangle=|\uparrow \uparrow\rangle, \\
& |2\rangle=\frac{1}{\sqrt{2}}(|\uparrow \downarrow\rangle+|\downarrow \uparrow\rangle), \quad|3\rangle=|\downarrow \downarrow\rangle,
\end{aligned}
$$

of singlet and triplet states on each rung, and construct a set of corresponding projection operators which generate the $\mathrm{su}(4)$ algebra

$$
\begin{gathered}
X_{i}^{\alpha \beta}=(|\alpha\rangle\langle\beta|)_{i}, \quad X_{i}^{\alpha \beta} X_{i}^{\delta \gamma}=\delta^{\beta \delta} X_{i}^{\alpha \gamma}, \\
\sum_{\alpha} X_{i}^{\alpha \alpha}=1, \quad\left[X_{i}^{\alpha \beta}, X_{j}^{\gamma \delta}\right]=\left(\delta^{\beta \gamma} X_{i}^{\alpha \delta}-\delta^{\alpha \delta} X_{j}^{\gamma \beta}\right) \delta_{i j},
\end{gathered}
$$

where $\alpha, \beta=0,1,2,3$. Spin operators on both chains of the ladder may be expressed in terms of this operator basis. ${ }^{22}$ The ladder Hamiltonian (1) is thus equivalent to a generalized, four-state chain with only nearest-neighbor interactions. It can be written as

$$
\begin{aligned}
H= & \sum_{i} \frac{1}{2} J_{\|}\left(P_{i, i+1}^{\|}-E_{i, i+1}^{-}\right)+\frac{1}{2} J_{\times}\left(P_{i, i+1}^{\times}-E_{i, i+1}^{+}\right) \\
& +\frac{1}{2} K\left(P_{i, i+1}^{\|}-E_{i, i+1}^{+}\right)-\left(\frac{1}{2} J_{\perp}+K\right)\left(X_{i}^{00}+X_{i+1}^{00}\right) \\
& +2 K X_{i}^{00} X_{i+1}^{00}+\frac{1}{2} K,
\end{aligned}
$$

where $P_{i, i+1}^{\|}$and $P_{i, i+1}^{\times}$are, respectively, permutation operators corresponding to ladder legs and plaquette diagonals, and the operators $E_{i, i+1}^{ \pm}$are unnormalized projectors on plaquette-singlet states. These operators satisfy simple algebraic relations. ${ }^{26}$ Their explicit expressions are

$$
P_{i, i+1}^{\|}=\sum_{\alpha, \beta=0}^{3} X_{i}^{\alpha \beta} X_{i+1}^{\beta \alpha},
$$

$$
P_{i, i+1}^{\times}=\sum_{\alpha, \beta=0}^{3}\left(1-2 \delta_{\alpha 0}\right)\left(1-2 \delta_{\beta 0}\right) X_{i}^{\alpha \beta} X_{i+1}^{\beta \alpha},
$$

and

$$
E_{i, i+1}^{ \pm}=2\left|\psi_{i, i+1}^{ \pm}\right\rangle\left\langle\psi_{i, i+1}^{ \pm}\right|,
$$

where the functions $\left|\psi_{i, i+1}^{ \pm}\right\rangle$are plaquette-singlet states, i.e., states with total spin per plaquette $S_{\square}^{2}=\left(\mathbf{S}_{1}+\mathbf{S}_{2}+\mathbf{S}_{3}+\mathbf{S}_{4}\right)^{2}$ $=0$, and may be expressed in terms of the rung states $|\alpha\rangle_{i}$ and $|\beta\rangle_{i+1}$ as

$$
\begin{aligned}
\left|\psi_{i, i+1}^{ \pm}\right\rangle= & \frac{1}{2}\left\{|0\rangle_{i}|0\rangle_{i+1} \pm|2\rangle_{i}|2\rangle_{i+1} \mp|1\rangle_{i}|3\rangle_{i+1}\right. \\
& \left.\mp|3\rangle_{i}|1\rangle_{i+1}\right\} .
\end{aligned}
$$

There are precisely two possible plaquette-singlet states, corresponding, respectively, to $E_{i, i+1}^{+}$and $E_{i, i+1}^{-}$. 


\section{B. Integrable models}

The complete set of exactly soluble isotropic ladder models with short-range interactions has been found in Ref. 22 using the algebraic Bethe-ansatz method (for a review see Ref. 30). Not all of these models are in the same class as that specified by Eq. (1), and here we present those cases relevant to a system with ring-exchange interactions. All of these are critical, and may be distinguished according to their central charge $c$, which characterizes the different universality classes close to integrable points.

$$
\text { 1. } c=2
$$
form

The exactly soluble models of this type have the simple

$$
H=\sum_{i}\left(P_{i}-E_{i}\right)
$$

where $P_{i}$ and $E_{i}$ denote, respectively, $P_{i, i+1}^{\|}, P_{i, i+1}^{\times}$and $E_{i, i+1}^{+}, E_{i, i+1}^{-}$. We thus obtain four different soluble Hamiltonians which correspond to the four combinations $\left\{P_{i}^{\|}, E_{i}^{+}\right\},\left\{P_{i}^{\|}, E_{i}^{-}\right\},\left\{P_{i}^{\times}, E_{i}^{+}\right\}$, and $\left\{P_{i}^{\times}, E_{i}^{-}\right\}$. Two of these are trivially soluble, because $\Sigma_{i}\left(P_{i}^{\|}-E_{i}^{-}\right)$is the Hamiltonian of two decoupled chains and $\Sigma_{i}\left(P_{i}^{\times}-E_{i}^{+}\right)$is the same pair of chains intertwined by the transformation $S_{2 i} \leftrightarrow T_{2 i}, \quad S_{2 i+1} \leftrightarrow S_{2 i+1}, \quad T_{2 i+1} \leftrightarrow T_{2 i+1} \quad$ [Eq. (5)]. The Hamiltonian of two decoupled chains is represented on the phase diagram of Fig. 2 by the point $J_{\|} / K=\infty$, with all other couplings equal to zero. Nonzero values of these couplings generally induce a spin gap, but because of the competing nature of different interaction terms there is a possibility of critical behavior for some combinations of these couplings. This will be demonstrated explicitly in Sec. IV. Of the remaining two solutions within the general ansatz (9), one is given by the combination $\left\{P_{i}^{\|}, E_{i}^{+}\right\}$, which in terms of spin operators is

$$
\begin{aligned}
H_{D}= & \sum_{i} \mathbf{S}_{i} \cdot \mathbf{S}_{i+1}+\mathbf{T}_{i} \cdot \mathbf{T}_{i+1}+\mathbf{S}_{i} \cdot \mathbf{T}_{i+1}+\mathbf{T}_{i} \cdot \mathbf{S}_{i+1} \\
& +4\left[\left(\mathbf{S}_{i} \cdot \mathbf{S}_{i+1}\right)\left(\mathbf{T}_{i} \cdot \mathbf{T}_{i+1}\right)-\left(\mathbf{S}_{i} \cdot \mathbf{T}_{i+1}\right)\left(\mathbf{S}_{i+1} \cdot \mathbf{T}_{i}\right)\right]
\end{aligned}
$$

The last $\left\{P_{i}^{\times}, E_{i}^{+}\right\}$is obtained by the same intertwining transformation from the previous Hamiltonian, and takes the form

$$
\begin{aligned}
H_{D I}= & \sum_{i} \mathbf{S}_{i} \cdot \mathbf{T}_{i+1}+\mathbf{T}_{i} \cdot \mathbf{S}_{i+1}+\mathbf{S}_{i} \cdot \mathbf{S}_{i+1}+\mathbf{T}_{i} \cdot \mathbf{T}_{i+1} \\
& +4\left[\left(\mathbf{S}_{i} \cdot \mathbf{T}_{i+1}\right)\left(\mathbf{T}_{i} \cdot \mathbf{S}_{i+1}\right)-\left(\mathbf{S}_{i} \cdot \mathbf{S}_{i+1}\right)\left(\mathbf{T}_{i} \cdot \mathbf{T}_{i+1}\right)\right]
\end{aligned}
$$

For all four massless models one obtains two decoupled Bethe-ansatz equations which correspond to the $D_{2}=\operatorname{su}(2)$ $\times \operatorname{su}(2)$ algebra. The energy of the Hamiltonian is the sum of two expressions for each SU(2) component, ${ }^{31}$ and thus the Hamiltonian (10) is equivalent to two decoupled chains. This model is critical (no spin gap), with a conformal charge $c$ $=2$. Equation (10) constitutes a part of the plaquette term, Eq. (2), while we will demonstrate explicitly below that the remaining contributions to $P_{4}+P_{4}^{-1}$ induce a spin gap, and therefore that the origin of coordinates in the phase diagram of Fig. 2 represents a gapped system. However, as shown in Sec. IV, the competition between different interaction terms of the Hamiltonian (1) drives the system to a second-order phase transition described by a CFT with $c=3 / 2$. The approximate form of this critical surface, represented in Fig. 2 by the shaded triangle, is obtained in Sec. IV.

Exact solutions in this class then provide two massless models, one in the limit of weak $K$ and one in the strong- $K$ limit, both of which correspond in the continuum limit to $c$ $=2$ WZW models. The four solutions of Eq. (9) are related by two transformations, one of which is the intertwining transformation shown above. The other is less transparent in nature, and is found by first noting that the two unnormalized projectors $E_{i}^{+}$and $E_{i}^{-}$are related by the $X$-operator transformation

$$
X_{k}^{0 a} \rightarrow-i X_{k}^{0 a}, \quad X_{k}^{a 0} \rightarrow i X_{k}^{a 0}
$$

for $a=1,2,3$ and $k=1, \ldots, N$, where $N$ is the total number of sites. This is a unitary transformation generated by the operator

$$
U(\pi / 2)=\exp \left[-i \frac{\pi}{2} \sum_{k=1}^{N}\left(X_{k}^{00}\right)\right]
$$

which has the property that

$$
H_{D}=U H_{2 c} U^{\dagger},
$$

where $H_{2 c}$ is the Hamiltonian of two uncoupled spin-1/2 Heisenberg chains. The nature of this transformation may be understood from the observation that it maps one of the plaquette-singlet state defined in Eq. (8) into the other, and thus that it transforms the projectors (7) according to $E_{i}^{+} \leftrightarrow E_{i}^{-}$. This transformation has the additional property that $[U(\pi / 2)]^{4}=1$, and therefore $U$ is one of the generators of the $Z_{4}$ transformation associated with the center of SU(4). In the following section we will demonstrate that this symmetry is essential for a complete understanding of the phase diagram.

$$
\text { 2. } c=1
$$

A second exact solution ${ }^{22}$ has the same eigenspectrum, but not the same degeneracies, as the spin-1/2 Heisenberg chain. The corresponding spin Hamiltonian

$$
\begin{aligned}
H_{1 / 2}= & -K \sum_{i}\left(\mathbf{S}_{i} \cdot \mathbf{T}_{i+1}+\mathbf{T}_{i} \cdot \mathbf{S}_{i+1}\right)+\frac{1}{2} K \sum_{\square}\left(P_{4}+P_{4}^{-1}\right) \\
& +J_{\perp} / 2 \sum_{i}\left(\mathbf{S}_{i} \cdot \mathbf{T}_{i}\right)
\end{aligned}
$$

commutes with the generators $\sum_{i} X_{i}^{a b}$ for any $a, b=1,2,3$, which form an su(3) subalgebra within the su(4) algebra generated by the $X$ operators. In view of completeness relation in 
Eq. (4), $H_{1 / 2}$ commutes with the total number operator $\Sigma_{i} X_{i}^{00}$ for singlets, and any multiple of this term may be added to the Hamiltonian without spoiling the integrability. The full symmetry of the model is thus $\mathrm{SU}(3) \times \mathrm{U}(1)=\mathrm{U}(3)$. In the framework of the equivalent Heisenberg chain, inspired by the Bethe-ansatz solution, the model may be considered as a chain of effective $\mathrm{SU}(2)$ "spins" $\mathbf{L}_{i}$ with

$$
L_{i}^{z} \equiv \frac{1}{2}-X_{i}^{00}=\frac{1}{4}-\mathbf{S}_{i} \cdot \mathbf{T}_{i} .
$$

The term $J_{\perp} / 2 K \sum_{i} \mathbf{S}_{i} \cdot \mathbf{T}_{i}$ in $H_{1 / 2}$ therefore corresponds to a coupling with a magnetic field $h \equiv J_{\perp} / 2 K{ }^{32}$ It is well known that the spin-1/2 chain in a magnetic field develops an incommensurate critical phase for $|h| \leqslant 2$ and has a massive phase for $|h|>2$ (see, for example, Ref. 33).

One observes from Eq. (15) that this integrable model corresponds to the line in parameter space $J_{\|}=0, J_{\times}=$ $-K$, for arbitrary $J_{\perp}$. When $\left(\left|J_{\perp} / K\right|\right) \leqslant 4$ the model is in the critical, incommensurate phase, whereas for $J_{\perp} / K>4$ the model has a gapped, rung-singlet phase, and in the region $J_{\perp} / K<-4$ it has a gapped, rung-triplet ground state. The nature of the gapped phases and the physical properties of the incommensurate phase will be discussed in more detail in Sec. III. In the critical region the model is described by a CFT with central charge $c=1 .{ }^{34}$ However, the presence of conserved charges generating the $\mathrm{U}(3)$ symmetry has the consequence $^{34}$ that the model possesses additional zero modes, and therefore while the conformal dimensions are those of the $c=1$ theory for the Coulomb gas, the degeneracies are altered accordingly. The critical region is represented on the phase diagram of Fig. 2 by the thick, dashed line AB, while the rung-singlet and ferromagnetic rung-triplet regions are denoted, respectively, by RS and FM. In Sec. IV (Fig. 10) we will demonstrate that there exists a finite critical region in the vicinity of the critical line.

$$
\text { 3. } c=3
$$

A further exact solution exists which corresponds simply to local Hamiltonians proportional to permutation operators $P_{i}^{\|}$and $P_{i}^{\times}$. The Hamiltonian corresponding to $P_{i}^{\|}$,

$$
H_{\|}=\sum_{i} \mathbf{S}_{i} \cdot \mathbf{S}_{i+1}+\mathbf{T}_{i} \cdot \mathbf{T}_{i+1}+4\left(\mathbf{S}_{i} \cdot \mathbf{S}_{i+1}\right)\left(\mathbf{T}_{i} \cdot \mathbf{T}_{i+1}\right)
$$

is invariant with respect to the full $\mathrm{SU}(4)$ group and describes a soluble "spin-orbital" model. ${ }^{35}$ In the continuum limit, Eq. (17) corresponds to an SU(4) WZW model at level $k=1,36,37$ and therefore has central charge $c=3$. This Hamiltonian commutes with the operator $\Sigma_{i}\left(\mathbf{S}_{i} \cdot \mathbf{T}_{i}\right)$, and thus is integrable for arbitrary values of $J_{\perp}$. One may also add a rung-rung coupling interaction to obtain the Hamiltonian

$$
H_{\|}^{\prime}=H_{\|}+J_{\perp} \sum_{i} \mathbf{S}_{i} \cdot \mathbf{T}_{i}+2 K \sum_{i}\left(\mathbf{S}_{i} \cdot \mathbf{T}_{i}\right)\left(\mathbf{S}_{i+1} \cdot \mathbf{T}_{i+1}\right),
$$

which was considered in Ref. 38 for arbitrary $J_{\perp}$ and for two values of $K(K=0$ and $K=-1)$. It was shown that for both

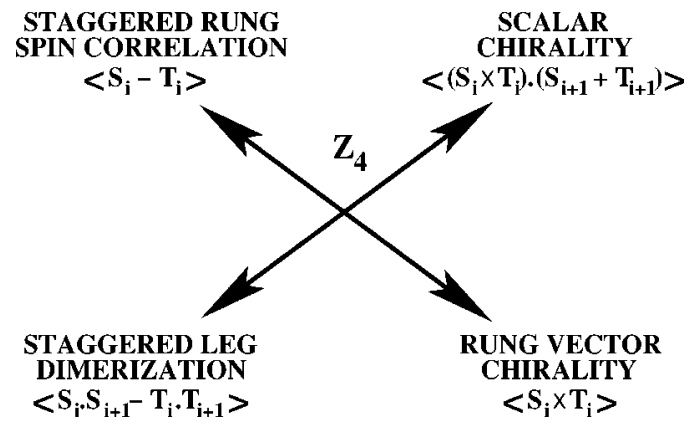

FIG. 3. Order parameters of the different phases related by the $Z_{4}$ transformation within the plane $J_{\|}-J_{\times}=K$.

values of $K$, three phases appear as a function of $J_{\perp}$, and are separated by two quantum critical points, $J_{+}^{c}$ and $J_{-}^{c}$. For $K=0$ and $J_{\perp}>J_{+}^{c}=2$ the model is in the rung-dimerized phase, for $J_{-}^{c}<J<J_{+}^{c}$ there is a gapless phase with $c=3$, and for $J<J_{-}^{c} \approx-1.79$ there is a gapless phase with $c=2$. For $K=-1$ the critical points occur at ${ }^{38} J_{+}^{c}=1 / 2$ and $J_{-}^{c} \approx$ -1.29 . Quite generally, the extent of the $c=3$ critical region is expected to be strongly reduced by a negative rung-rung coupling, while it will expand for positive $K$. The critical behavior of $H_{\|}^{\prime}$ is thus expected to persist for finite regions of parameter space, and the position of the critical points to depend strongly on the rung-rung coupling. We emphasize that the model of Eq. (18) does not appear in the phase diagram of the ladder model under consideration (1) but we have introduced its properties here for use in Sec. IV as one appropriate basis model for a perturbative expansion. Similar considerations apply to $H_{\times}$, which is obtained from $H_{\|}$by using the intertwining transformation of Sec. II B 1.

\section{EXACT GROUND STATES}

\section{A. $Z_{4}$ plane}

In this section we apply certain discrete symmetry considerations to obtain further insight into the structure of the phase diagram. We employ the matrix-product ansatz to find exact ground states, and also the boundaries between these, even in the region of parameter space where the Hamiltonian (1) is not exactly integrable.

We begin by noting that for arbitrary $J_{\perp}$, when

$$
J_{\|}-J_{\times}=K,
$$

the Hamiltonian (1) is invariant with respect to the $Z_{4}$ transformation (13) which generates the mapping $E_{i}^{ \pm} \rightarrow E_{i}^{+}$. The condition (19) defines a plane in the 3D space of the phase diagram, marked in Fig. 2 by parallel dot-dashed lines. The Hamiltonian of the system in the $Z_{4}$-symmetric plane commutes with the singlet total number operator $\sum_{i} X_{i}^{00}$.

This $Z_{4}$ transformation is precisely the one noted in Refs. 15 and 39, in which it was referred to as a duality transformation. It is a canonical transformation for the lowest $(s$ $=1 / 2 \times s=1 / 2)$ representation of $\mathrm{SU}(2) \times \mathrm{SU}(2)$, and conserves the values of the Casimir operators in this representation. In terms of the original spin variables it takes the form 


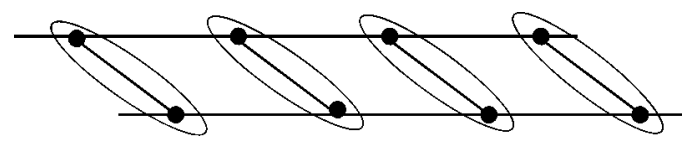

FIG. 4. Representation of the rung-singlet (RS) phase. The ellipses denote a singlet state of the spins on each rung.

$$
\begin{aligned}
& \widetilde{\mathbf{S}}_{i}=\frac{1}{2}\left(\mathbf{S}_{i}+\mathbf{T}_{i}\right)-\mathbf{S}_{i} \times \mathbf{T}_{i}, \\
& \widetilde{\mathbf{T}}_{i}=\frac{1}{2}\left(\mathbf{S}_{i}+\mathbf{T}_{i}\right)+\mathbf{S}_{i} \times \mathbf{T}_{i} .
\end{aligned}
$$

When expressed using the variables $\widetilde{\mathbf{S}}_{i}, \widetilde{\mathbf{T}_{i}}$, the Hamiltonian $H_{D}$ (10) is exactly that of two decoupled spin chains. It is clear from the definition (2) of the plaquette term that $H_{D}$ constitutes a part of the operator $P_{4}+P_{4}^{-1}$, while the remaining contributions to this term couple the two effective spin$1 / 2$ chains to produce a system with a finite spin gap.

In the $Z_{4}$ plane this transformation acts as a symmetry between different order parameters. It maps the order parameter $\left\langle\mathbf{S}_{i}-\mathbf{T}_{i}\right\rangle$ for antiferromagnetic rung spin correlations into the vector-chirality order parameter $\left\langle\mathbf{S}_{i} \times \mathbf{T}_{i}\right\rangle$ $\equiv\left\langle\widetilde{\mathbf{S}}_{i}-\widetilde{\mathbf{T}}_{i}\right\rangle$, while the leg dimer order parameter $\left\langle\mathbf{S}_{i} \cdot \mathbf{S}_{i+1}\right.$ $\left.-\mathbf{T}_{i} \cdot \mathbf{T}_{i+1}\right\rangle$ is mapped into the order parameter $\left\langle\left(\mathbf{S}_{i}+\mathbf{T}_{i}\right) \cdot\left(\mathbf{S}_{i+1} \times \mathbf{T}_{i+1}\right)+\left(\mathbf{S}_{i} \times \mathbf{T}_{i}\right) \cdot\left(\mathbf{S}_{i+1}+\mathbf{T}_{i+1}\right)\right\rangle$ for scalar chirality (Fig. 3). Phases characterized by order parameters related under the $Z_{4}$ transformation within each pair are therefore symmetric within the plane defined by Eq. (19). The point $J_{\perp}=J_{\|}=K$ considered in the DMRG studies of Refs. 15 and 16 lies in this plane (the star in Fig. 2), giving the properties of duality observed in the results of both analyses.

From this consideration we may deduce the nature of a candidate ground state as a product of linear superpositions of the two plaquette-singlet states. The complete set of plaquette states with fixed angular momentum contains one quintuplet $(j=2)$, three triplets $(j=1)$, and two singlets $(j$ $=0)$. The two plaquette-singlet states are those constructed above, the states with $j=1$ are created by applying a linear combination of operators $P_{i, i+1}^{\|}, P_{i, i+1}^{\times}$, and $X_{i}^{00} X_{i+1}^{00}$, and the state with $j=2$ is created by the action of a combination of the operators $P_{i, i+1}^{\|}, P_{i, i+1}^{\times}$, and $E_{i, i+1}^{ \pm}$. The expressions for these states allow their identification with matrixproduct-ansatz states, while the explicit form of the Hamiltonian (5) corresponds to the operator basis for matrixproduct-ansatz Hamiltonians. ${ }^{40}$ One observes that in the $Z_{4}$-symmetric plane, $J_{\|}-J_{\times}=K$, the condition $\left(J_{\perp} / K\right)>4$ defines a region in the phase diagram (Fig. 2) with an exact rung-singlet $(\mathrm{RS})$ ground state,

$$
\left|\psi_{R S}\right\rangle=|0\rangle_{1}|0\rangle_{2} \cdots|0\rangle_{N-1}|0\rangle_{N},
$$

which is represented schematically in Fig. 4. On the line

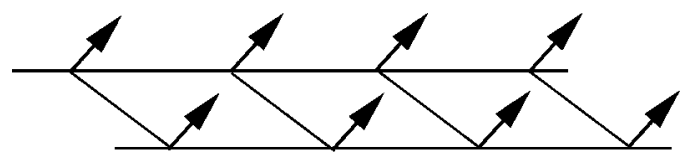

FIG. 5. Typical ferromagnetic (FM) ground state for large, negative $J_{\perp}$.

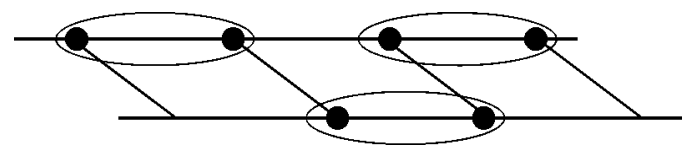

FIG. 6. Staggered leg-dimerized (SD) state.

$J_{\times}=-K, J_{\|}=0$, which lies in the $Z_{4}$-symmetric plane, this statement is supported by the exact solution of the Hamiltonian $H_{1 / 2}(15)$ of the preceding section. From the results of Ref. 40 , the line $J_{\perp} / K=4$ in the $Z_{4}$-symmetric plane is a line of second-order phase transitions into a spontaneously dimerized phase of staggered leg dimerization.

Similarly, the condition $J_{\perp} / K<-4$ defines an exact ferromagnetic $(\mathrm{FM})$ rung ground state,

$$
\left|\psi_{F M}\right\rangle=X_{1}^{a 0} X_{2}^{a 0} \cdots X_{N-1}^{a 0} X_{N}^{a 0}|0\rangle,
$$

in which $|0\rangle$ denotes the global state of singlets on every rung. This state is represented in Fig. 5. The transition on the line $J_{\perp} / K=-4$ is of first order. Both transition lines are determined exactly from the points where the dispersion relations of the elementary excitations become massless. In the rung-singlet phase this excitation is a propagating rung triplet, while in the ferromagnetic phase it is a rung singlet. The second-order transition from the rung-singlet phase to the staggered dimer phase (Fig. 6) lies in the universality class of the spin-1 bilinear-biquadratic chain, which in the continuum limit is described by a $c=3 / 2 \mathrm{CFT}^{23}$ and is a transition of the Babujian-Takhtajan type. This transition is characterized by a spontaneous breaking of discrete $Z_{2}$ symmetry, which in the ladder is related to translation by one lattice unit.

From the $Z_{4}$ duality of the system we deduce that at large $K$ (near the origin of Fig. 2) there is another second-order phase transition of the same universality class from a scalarchirality phase (Fig. 7), which is dual to the staggered dimer phase, to a vector-chirality phase (Fig. 8), which is dual to the rung-singlet phase. In the following section we provide further arguments in support of this statement. Figures 7 and 8 show, respectively, typical static configurations of spins in scalar- and vector-chirality phases; these should be understood only as indicating the preferred instantaneous spin configurations, which in fact fluctuate rapidly, such that the phases exist only in the sense of finite average values of the corresponding spin-correlation functions. All four phases have only short-ranged correlations in the chain direction. These states illustrate the increasing dominance of the $K$ term, which favors configurations in which all spins on a plaquette are mutually perpendicular to maximize their solid angle, ${ }^{11}$ in the competition with nearest-neighbor exchange terms which favor antiparallel spins.

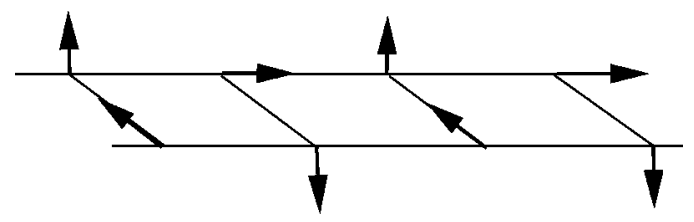

FIG. 7. Representation of a spin configuration with finite scalarchirality correlation function. 


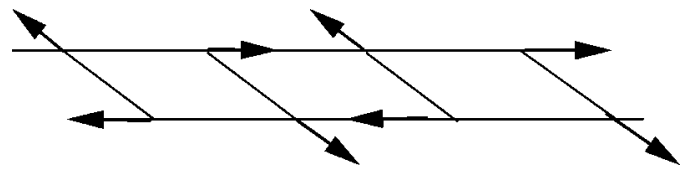

FIG. 8. Schematic representation of a static vector-chirality configuration.

Finally, the first-order phase transition from the ferromagnetic phase takes the system to a type of Affleck-KennedyLieb-Tasaki (AKLT) state ${ }^{41}$ formed by effective spin-1 variables represented by the rung triplets (Fig. 9). It differs from the ferromagnetic phase in that coherence between the individual rung triplets is not established until the transition at $J_{\perp} / K=-4$. This state is obtained directly from the vectorchirality phase in a first-order transition at small, negative $J_{\perp}$. We stress that the letters indicating the locations of these phases in Fig. 2 should be understood to refer only to the plane of exact $Z_{4}$ symmetry, including the rung-singlet and ferromagnetic phases which lie outside the region marked by the dot-dashed lines. However, because there are no phase transitions other than those present on the diagram, the gapped phases obtained for model parameters outside the plane are connected continuously to those of the exactly known states, and their physical properties evolve continuously as the values of the interactions are further changed away from the $Z_{4}$ plane.

\section{B. Incommensurate line}

With these results, a heuristic understanding of the origin of the incommensurate phase appearing on the critical line $\mathrm{AB}$ in Fig. 2 (Sec. II) may be obtained by considering the role of $J_{\perp}$ as an effective magnetic field. We stress first that the incommensurate phase arises with no breaking of SU(2) symmetry in the space of the spin variables $\mathbf{S}_{i}$ and $\mathbf{T}_{i}$. The variable conjugate to the effective field, $\Sigma_{i} \mathbf{S}_{i} \cdot \mathbf{T}_{i}$, corresponds [Eq. (16)] to an average singlet density which varies from 0 at the boundary to the ferromagnetic phase $\left(J_{\perp}\right.$ $\rightarrow-4 K)$ to 1 at the rung-singlet phase boundary $\left(J_{\perp}\right.$ $\rightarrow 4 K$ ), in exact analogy to $\sum_{i} S_{i}^{z}$ for the spin-chain problem. For the ladder, $\Sigma_{i} \mathbf{S}_{i} \cdot \mathbf{T}_{i}$ also expresses the spin correlation on each rung, which may be characterized by defining a variable $k_{\perp}=\cos ^{-1}\left(\left\langle\mathbf{S}_{i} \cdot \mathbf{T}_{i}\right\rangle /\left|\mathbf{S}_{i}\right|\left|\mathbf{T}_{i}\right|\right)$. This effective wave vector across the ladder varies continuously from 0 to $\pi$ over the range $-4 K \leqslant J_{\perp} \leqslant 4 K$, and takes the value $k_{\perp}=\pi / 2$ at $J_{\perp}$ $=0$, where the ring-exchange term, favoring locally perpendicular spins, is dominant.

The incommensurate state in the ladder direction is crucially dependent on the parameters of the $c=1$ line, notably the special role of the $J_{\times}$term. Only for $J_{\times}=-K$ is the spectrum massless, with quasi-long-ranged correlation func-

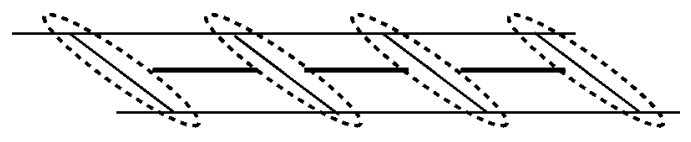

FIG. 9. AKLT-type ground state. The dashed ellipses represent effective spin-1 variables formed by triplet states on the ladder rungs. tions classifiable by a ladder wave vector $q$ which reflect the competition between nearest-neighbor exchange and ring exchange, again in exact analogy with the spin-1/2 Heisenberg chain in a magnetic field. ${ }^{42}$ The nature of the incommensurate correlations arising from the excitation spectrum is elucidated by considering the effective spin variables $\mathbf{L}_{i}$. The analogous "spin" correlation function is given by

$$
\left\langle\mathbf{L}_{i} \cdot \mathbf{L}_{j}\right\rangle=\left\langle X_{i}^{0 a} X_{j}^{a 0}+X_{i}^{a 0} X_{j}^{0 a}\right\rangle+2\left\langle\left(\frac{1}{2}-X_{i}^{00}\right)\left(\frac{1}{2}-X_{j}^{00}\right)\right\rangle,
$$

where the first line corresponds to terms of the form $L_{i}^{+} L_{j}^{-}$ $+L_{i}^{-} L_{j}^{+}$and the second to $L_{i}^{z} L_{j}^{z}$. In terms of the original spin variables one obtains

$$
\begin{aligned}
\left\langle\mathbf{L}_{i} \cdot \mathbf{L}_{j}\right\rangle= & \frac{1}{2}\left\langle(\mathbf{S}-\mathbf{T})_{i} \cdot(\mathbf{S}-\mathbf{T})_{j}\right\rangle+2\left\langle(\mathbf{S} \times \mathbf{T})_{i} \cdot(\mathbf{S} \times \mathbf{T})_{j}\right\rangle \\
& +\frac{1}{2}\left\langle\left(\frac{1}{2}+2 \mathbf{S}_{i} \cdot \mathbf{T}_{i}\right)\left(\frac{1}{2}+2 \mathbf{S}_{j} \cdot \mathbf{T}_{j}\right)\right\rangle
\end{aligned}
$$

Thus the dynamical correlation function exhibiting a peak at the incommensurate wave vector $q$ is in fact the sum of the staggered rung, vector chirality, and singlet density correlation functions, although from the massless nature of the excitations at $q$ one may expect peaks in each function individually. Again the terms in the first line correspond to $L_{i}^{+} L_{j}^{-}+L_{i}^{-} L_{j}^{+}$, and are symmetrical under the $Z_{4}$ transformation (Fig. 3) in the plane: their peaks appear at the same wave vector for all values of $J_{\perp}$, but their intensities may differ. The effective $L_{i}^{z} L_{j}^{z}$ terms (second line) are not symmetrical with the others for all couplings $J_{\perp} \neq 0$ (finite effective field), but as in the spin chain may be expected to show the same continuous evolution of the incommensurate peak position from $q=0$ at $J_{\perp}=-4 K$ to $q=\pi$ at $J_{\perp}=0$, and back to $q=0$ at $J_{\perp}=4 K$. We stress again that this behavior is a specific property of the incommensurate line and cannot in general be expected to be clearly visible in other parts of the phase diagram not in its immediate vicinity, including the regions corresponding to DMRG analyses performed to date. However, in Sec. IV we isolate a region in which the incommensurate properties (albeit for a gapped excitation spectrum) may persist over a significant range of parameters.

\section{RENORMALIZATION-GROUP ANALYSIS}

In this section we study the perturbations around different CFT solutions, which either appear in the phase diagram of the model (1) or are closely related to it. We adopt a variety of techniques to address the nature of the ground states in regions away from these soluble points, and to establish the fixed-point structure of the phase diagram. Because all of the transition points and lines are known, the relevance of the operators within Eq. (1) as perturbations of the exact solutions determines the flow under renormalization and thus the dominant physical properties of the intermediate regions. In each section and in the Appendix, known results are summarized briefly while those which are new in the current context are presented in detail.

We have found four models suitable for this type of analysis. From the exact solutions of Sec. II, there are two CFTs with $c=2$ which correspond to two decoupled chains de- 
scribed by the soluble Hamiltonian of Eq. (10); one solution is relevant for weak $K$ and the other for strong $K$. Here we concentrate primarily on the strong-coupling regime, i.e., large $K$, for which significantly less is known: the fermionized version of the limiting model of two effectively decoupled spin chains is analyzed in Sec. IV A by a conventional RG procedure. We have argued from the presence of the $Z_{4}$ symmetry (Secs. II and III) that in the strong-coupling limit there is a second-order phase transition with $c=3 / 2$, represented by the shaded triangle on the phase diagram of Fig. 2 . The Zamolodchikov $c$ theorem ${ }^{24}$ then demands that weakand strong-coupling limits are disjoint in RG sense, i.e., there is no continuous flow from weak to strong coupling. To describe the intermediate regime between the two second-order phase transitions with $c=3 / 2$ we therefore use in Sec. IV B another CFT with $c=3$, which is described by the model (18). By perturbative analysis of a fermionized model we find that this intermediate region is gapped; an alternative perturbative treatment of the SU(4) model (17) has recently provided similar results. ${ }^{39}$ Finally, the same approach may also be applied to the $c=1$ CFT which corresponds to the solution (15). In the vicinity of the line $A B$ on the phase diagram of Fig. 2, we employ rather general arguments in Sec. IV C to reveal the presence of a massless region in one plane of the phase diagram. The CFTs with $c=3 / 2$ thus represent unstable points in parameter space and must be accompanied by a flow towards stable fixed points. Based on the arguments of Ref. 23 the stable theory should be a $\mathrm{SU}(2)_{k=1}$ CFT with $c=1$, the natural candidate for which is the CFT corresponding to the model of Eq. (15).

\section{A. $c=2$ CFTs}

A weak-coupling bosonization analysis is appropriate for the limit of two quasidecoupled chains when $J_{\|} \gg K$ and $J_{\|}$ $\gg J_{\perp}$. The ladder system with biquadratic exchange has been shown to undergo a second-order phase transition at which the behavior of the massless modes is governed by a CFT with central charge $c=3 / 2 .{ }^{43}$ The consideration of a ringexchange term is technically identical (see Ref. 10), and the results are found to be in good agreement with those from other approaches.

When performing a perturbative analysis around CFTs, all contributions to the spin Hamiltonian should be classified according to the scaling dimensions of the operator content of the corresponding CFT. In the case of perturbations around the limit of two decoupled chains, in both weak- and strong- $K$ limits, four-spin interaction terms and interleg couplings are expressed in terms of two fundamental $c=1$ WZW fields, $g_{a}$ and $g_{a}^{\prime}(a=0,1,2,3)$, with conformal dimension $\left(\frac{1}{4}, \frac{1}{4}\right)$, and of Kac-Moody currents $\mathbf{J}$ and $\overline{\mathbf{J}}$ with dimensions $(1,0)$ and $(0,1)$. This theory can be further expressed in terms of four different Ising models, ${ }^{44,43}$ i.e., of orderdisorder fields, energy operators, and Majorana fermions. The representation of these fields by four Ising models is summarized in the Appendix. The connection to the spin variables is given by

$$
\widetilde{\mathbf{S}}_{i} \rightarrow a \widetilde{\mathbf{S}}(x),
$$

$$
\begin{gathered}
\widetilde{\mathbf{S}}(x)=\mathbf{J}+\overline{\mathbf{J}}+(-1)^{x / a} \Theta \operatorname{Tr}[g(x) \sigma], \\
\widetilde{\mathbf{T}}(x)=\mathbf{J}^{\prime}+\overline{\mathbf{J}}^{\prime}+(-1)^{x / a} \Theta \operatorname{Tr}\left[g^{\prime}(x) \sigma\right] .
\end{gathered}
$$

We note that in Eq. (25) the uniform and staggered parts of the spin-density operators have different conformal dimensions, and that $\Theta$ is a nonuniversal normalization constant.

The expression in terms of Ising-model fermions for the general ladder Hamiltonian, which includes arbitrary leg-leg, diagonal-diagonal, and rung-rung couplings, is derived in the Appendix, and is valid around the limits of two decoupled chains. There are two such limits, the weak- $K$ regime which corresponds to the two initial chains and the strong- $K$ regime which corresponds to two $Z_{4}$-rotated chains (14), resulting in the Hamiltonian (10). The expressions of the Appendix are valid for both cases.

The resulting continuum-limit Hamiltonian for the system in weak- and strong-coupling regimes may be expressed in terms of four Majorana fermions with different singlet and triplet masses, ${ }^{43}$

$$
\begin{aligned}
H= & \frac{-i}{2} \int d x\left[v_{s}\left(\psi^{0} \partial_{x} \psi^{0}-\bar{\psi}^{0} \partial_{x} \bar{\psi}^{0}\right)+m_{s} \psi^{0} \bar{\psi}^{0}\right. \\
& \left.+\sum_{a=1,2,3} v_{t}\left(\psi^{a} \partial_{x} \psi^{a}-\bar{\psi}^{a} \partial_{x} \bar{\psi}^{a}\right)+m_{t} \psi^{a} \bar{\psi}^{a}\right]+H_{\text {marg }}
\end{aligned}
$$

The Hamiltonian of the marginal interactions,

$$
\begin{gathered}
H_{\mathrm{marg}}=\int d x\left[\lambda_{1} O_{1}+\lambda_{2} O_{2}\right], \\
O_{1}=\psi^{1} \bar{\psi}^{1} \psi^{2} \bar{\psi}^{2}+\psi^{1} \bar{\psi}^{1} \psi^{3} \bar{\psi}^{3}+\psi^{2} \bar{\psi}^{2} \psi^{3} \bar{\psi}^{3}, \\
O_{2}=\psi^{0} \bar{\psi}^{0}\left(\psi^{1} \bar{\psi}^{1}+\psi^{2} \bar{\psi}^{2}+\psi^{3} \bar{\psi}^{3}\right),
\end{gathered}
$$

contains current-current contributions of the forms $\left(\mathbf{J}_{1}\right.$ $\left.+\overline{\mathbf{J}}_{1}\right) \cdot\left(\mathbf{J}_{2}+\overline{\mathbf{J}}_{2}\right)$, arising from the interleg interactions, and $-\left(\mathbf{J}_{1} \overline{\mathbf{J}}_{1}+\mathbf{J}_{2} \overline{\mathbf{J}}_{2}\right)$ from the intraleg couplings, as well as a contribution from the normal-ordered marginal product $\operatorname{Tr}\left(\sigma^{a} g\right) \operatorname{Tr}\left(\sigma^{a} g^{\prime}\right) \operatorname{Tr}\left(\sigma^{b} g\right) \operatorname{Tr}\left(\sigma^{b} g^{\prime}\right)$, which originates in the four-spin term $\left(\widetilde{\mathbf{S}}_{i} \cdot \widetilde{\mathbf{T}}_{i}\right)\left(\widetilde{\mathbf{S}}_{i+1} \cdot \widetilde{\mathbf{T}}_{i+1}\right)$. Explicit expressions for the marginal couplings $\lambda_{1}$ and $\lambda_{2}$ are given in the Appendix.

Weak Coupling. In the weak-coupling regime the results of the Appendix provide the expressions

$$
\begin{gathered}
m_{t}=J_{\perp}-2 J_{\times}-20 \lambda^{2} K, \\
m_{s}=-3 J_{\perp}+6 J_{\times}+12 \lambda^{2} K,
\end{gathered}
$$

for triplet and singlet masses, where $\lambda$ is another nonuniversal quantity. The marginal interactions renormalize these masses, and the phase transition occurs when the renormalized triplet mass vanishes, i.e., $m_{t}^{\mathrm{ren}}=0$. On the other hand, the matrix-product ansatz (Sec. III) gives the phase-transition line exactly. One may therefore attempt to specify the renormalization by taking (for example, at $J_{\times}=0$ ) the value of $\lambda^{2}$ 
to be in agreement with the exact result, which suggests that $\lambda^{2}=1 / 5$ for this second-order phase transition.

Strong Coupling. In the strong-coupling regime we analyze perturbations around the CFT which correspond to the exact solution of Eq. (10). For this it is convenient to perform the transformation (20), which from Eq. (1) yields

$$
\begin{aligned}
H= & \sum_{i} \frac{1}{2} K\left(\widetilde{\mathbf{S}}_{i} \cdot \widetilde{\mathbf{S}}_{i+1}+\widetilde{\mathbf{T}}_{i} \cdot \widetilde{\mathbf{T}}_{i+1}\right)+\left(J_{\perp}+K\right)\left(\widetilde{\mathbf{S}}_{i} \cdot \widetilde{\mathbf{T}}_{i}\right) \\
& +\frac{1}{2} J_{\times\{}\left\{\widetilde{\mathbf{S}}_{i} \cdot \widetilde{\mathbf{T}}_{i+1}+\widetilde{\mathbf{T}}_{i} \cdot \widetilde{\mathbf{S}}_{i+1}+\widetilde{\mathbf{S}}_{i} \cdot \widetilde{\mathbf{S}}_{i+1}+\widetilde{\mathbf{T}}_{i} \cdot \widetilde{\mathbf{T}}_{i+1}\right. \\
& \left.-4\left[\left(\widetilde{\mathbf{S}}_{i} \cdot \widetilde{\mathbf{S}}_{i+1}\right)\left(\widetilde{\mathbf{T}}_{i} \cdot \widetilde{\mathbf{T}}_{i+1}\right)-\left(\widetilde{\mathbf{S}}_{i} \cdot \widetilde{\mathbf{T}}_{i+1}\right)\left(\widetilde{\mathbf{S}}_{i+1} \cdot \widetilde{\mathbf{T}}_{i}\right)\right]\right\} \\
& +\frac{1}{2} J_{\|}\left\{\widetilde{\mathbf{S}}_{i} \cdot \widetilde{\mathbf{S}}_{i+1}+\widetilde{\mathbf{T}}_{i} \cdot \widetilde{\mathbf{T}}_{i+1}+\widetilde{\mathbf{S}}_{i} \cdot \widetilde{\mathbf{T}}_{i+1}+\widetilde{\mathbf{T}}_{i} \cdot \widetilde{\mathbf{S}}_{i+1}\right. \\
& \left.+4\left[\left(\widetilde{\mathbf{S}}_{i} \cdot \widetilde{\mathbf{S}}_{i+1}\right)\left(\widetilde{\mathbf{T}}_{i} \cdot \widetilde{\mathbf{T}}_{i+1}\right)-\left(\widetilde{\mathbf{S}}_{i} \cdot \widetilde{\mathbf{T}}_{i+1}\right)\left(\widetilde{\mathbf{S}}_{i+1} \cdot \widetilde{\mathbf{T}}_{i}\right)\right]\right\} \\
& +2 K \sum_{i}\left(\widetilde{\mathbf{S}}_{i} \cdot \widetilde{\mathbf{T}}_{i}\right)\left(\widetilde{\mathbf{S}}_{i+1} \cdot \widetilde{\mathbf{T}}_{i+1}\right) .
\end{aligned}
$$

By substituting

$$
\begin{gathered}
J_{L}=K / 2, \\
J_{D}=J_{\times} / 2+J_{\|} / 2, \\
V_{L L}=-2 J_{\times}+2 J_{\|}, \\
V_{D D}=2 J_{\times}-2 J_{\|}, \\
V_{R R}=2 K, \\
J_{R}=J_{\perp}+K,
\end{gathered}
$$

in Eq. (A9) the expressions for triplet and singlet masses become

$$
\begin{gathered}
m_{t}=J_{\perp}+K-J_{\times}-J_{\|}-\lambda^{2}\left(16 J_{\|}+4 K-16 J_{\times}\right), \\
m_{s}=-3 J_{\perp}-3 K+3 J_{\times}+3 J_{\|}+12 \lambda^{2} K .
\end{gathered}
$$

The marginal current-current interactions act again to renormalize these masses. Setting $J_{\times}=0$ and taking $J_{\perp}=J_{\|}=J$ yields the value of the second-order phase-transition point as $K / J=16 \lambda^{2} /\left(1-4 \lambda^{2}\right)$. A comparison with the DMRG results of Ref. 16, which suggest that at strong coupling the transition point is $K / J \approx 5-6$ [in the units of Eq. (1)], yields $\lambda^{2}$ $\approx 1 / 7$, a value rather close to that obtained in the weakcoupling regime. After this the equation $m_{t}=0$ gives the form of the critical surface in the strong-coupling limit, which is represented by the shaded triangle in the phase diagram of Fig. 2 and by the bold, dashed line in Fig. 10; the shaded triangle lies below the $Z_{4}$-symmetric plane, but does not include the origin of the coordinate systems. We note here that the perturbative considerations applied above are in a strict sense questionable, because in the problem under consideration the "perturbative" rung-rung coupling is of the same order as the coupling corresponding to the unperturbed CFT, which is $K / 2$. For a ladder system in which $V_{R R}$ is not fixed to be $2 K$, and is small in comparison with $V_{L L}$ and $V_{D D}$, the second-order phase transition with central charge $c=3 / 2$ in the strong-coupling limit is accessible by perturbative analysis around the exact solution (10). Thus for consistency this term should be added to the Hamiltonian with a small coupling constant, $V_{R R}$, in order to study the RG behavior. We remark also that for $J_{\times}=J_{\|} \equiv J$, the leg-leg and diagonal-diagonal biquadratic terms in Eq. (29) cancel explicitly and the effective theory is described by massive fermions with a singlet-triplet mass splitting generated by $V_{R R}$ and $J_{R}$.

The lines of phase transitions correspond to the vanishing of the triplet mass $m_{t}$. The theory is then equivalent to three massless fermions and is therefore described by a $c=3 / 2$ WZW model. Because of the SU(2) symmetry of the ladder model (1), this is a SU(2) WZW model at level $k=2$. The bare singlet and triplet masses acquire a renormalization due to marginal interactions which can in principle be computed from the RG analysis. By considering the operator product expansion (OPE) for operators $O_{1}, O_{2}, \psi_{0} \bar{\psi}_{0}$, and $\psi_{a} \bar{\psi}_{a}$ $(a=1,2,3)$ [see Appendix and Eqs. (25) and (27)], we deduce the one-loop RG equations

$$
\begin{gathered}
\frac{d \lambda_{1}}{d(\ln L)}=2 \pi\left(\lambda_{1}^{2}+\lambda_{2}^{2}\right), \quad \frac{d \lambda_{2}}{d(\ln L)}=4 \pi \lambda_{1} \lambda_{2}, \\
\frac{d g_{s}}{d(\ln L)}=g_{s}+3 \pi g_{t} \lambda_{2}, \\
\frac{d g_{t}}{d(\ln L)}=g_{t}\left(1+2 \pi \lambda_{1}\right)+\pi g_{s} \lambda_{2},
\end{gathered}
$$

for the marginal couplings $\lambda_{1}$ and $\lambda_{2}$, and for the singlet and triplet couplings $g_{s}$ and $g_{t}$, which are, respectively, proportional to $-m_{s}$ and $m_{t}$. A similar analysis is performed in Refs. 43 and 44). The first and the second equations are decoupled from the others, and can be integrated [in variables $\left.\lambda_{ \pm}=\frac{1}{2}\left(\lambda_{1} \pm \lambda_{2}\right)\right]$ to yield y $^{44}$

$$
\lambda_{ \pm}=\frac{\lambda_{ \pm}^{(0)}}{1-8 \pi \lambda_{ \pm}^{(0)} \ln \left(L / L_{0}\right)} .
$$

From this it follows that for negative initial $\lambda_{ \pm}$these couplings are marginally irrelevant (and renormalize to 0 ), whereas for positive values they are marginally relevant (and develop exponential gaps). The initial (ultraviolet) fixed point is given by $g_{s}=g_{t}=0$, which corresponds to the CFT with $c=2$ (two uncoupled chains), and the RG equations for $g_{s}$ and $g_{t}$ yield the renormalization of masses under the RG flow. The new unstable fixed point is then defined from the renormalized value of $m_{t}$ as $m_{t}^{*}=0$. Because of these renormalization effects the exact transition line is difficult to estimate, but the presence of this transition is to be expected from $Z_{4}$-symmetry arguments.

Self-dual model. From the analysis above one may conclude that the lines (or surfaces) of the two second-order phase transitions with $c=3 / 2$ are not $Z_{4}$ symmetric, because the rung-rung coupling is proportional to $K$. The Hamiltonian which would have perfect symmetry of the two second-order phase transitions is 


$$
\begin{aligned}
H= & J_{\|} \sum_{i}\left(\mathbf{S}_{i} \cdot \mathbf{S}_{i+1}+\mathbf{T}_{i} \cdot \mathbf{T}_{i+1}\right)+J_{R} \sum_{i}\left(\widetilde{\mathbf{S}}_{i} \cdot \widetilde{\mathbf{T}}_{i}\right), \\
& +\frac{1}{2} K \sum_{i}\left(\widetilde{\mathbf{S}}_{i} \cdot \widetilde{\mathbf{S}}_{i+1}+\widetilde{\mathbf{T}}_{i} \cdot \widetilde{\mathbf{T}}_{i+1}\right)+V_{R R} \sum_{i}\left(\widetilde{\mathbf{S}}_{i} \cdot \widetilde{\mathbf{T}}_{i}\right) \\
& \times\left(\widetilde{\mathbf{S}}_{i+1} \cdot \widetilde{\mathbf{T}}_{i+1}\right),
\end{aligned}
$$

which corresponds to the model with $J_{R}$ and $V_{R R}$ independent of $K$. In the small- $J_{\|}$limit we have obtained two SU(2) WZW models perturbed by relevant and marginal interactions which have their origin in the $K$ term and in the small couplings $J_{R}$ and $V_{R R}$. In the strong- $K$ regime there are again two SU(2) WZW models perturbed by the small $J_{\|}$, $J_{R}$, and $V_{R R}$ terms. We note that this Hamiltonian may be considered as a two-chain Hamiltonian $\Sigma_{i} P_{i}^{\|}-E_{i}^{-}$perturbed by terms proportional to $P_{i}^{\|}-P_{i}^{\times}$, or as a $Z_{4}$-transformed Hamiltonian with the $Z_{4}$-transformed perturbation. Qualitatively, the resulting behavior is the same in both cases. In the continuum limit this perturbation is represented by two fourfermion terms [see Eq. (41)], one of which gives the marginal contribution while the other gives the relevant contribution.

Intermediate coupling. From the Zamolodchikov $c$ theorem, ${ }^{24}$ a CFT perturbed by relevant and marginally relevant interactions will flow either to another CFT with smaller central charge or to a massive phase. For small $K$ and $J_{\perp}$, perturbation around the limit of decoupled chains generates a flow to a $c=3 / 2 \mathrm{CFT}$ which is in the universality class of the bilinear-biquadratic $S=1$ spin chain, a result established recently in Refs. 10 and 13. In the strongcoupling limit (large $K$ ) one may consider the $J_{\|}$term as a small perturbation and the same arguments are applicable (but now for the variables $\widetilde{\mathbf{S}}$ and $\widetilde{\mathbf{T}}$ ), the corresponding RG flow being from a critical $c=2 \mathrm{CFT}$ to a $c=3 / 2 \mathrm{CFT}$. The universality class of this transition is the same as above: it is described by an SU(2)-symmetric CFT with level- $k=2 \mathrm{Kac}-$ Moody algebra.

From this one may conclude that the weak-coupling regime is not related continuously to the strong-coupling regime, and that there is a crossover between the two. The natural candidate for this crossover region would be the $Z_{4}$-symmetric plane, $J_{\|}-J_{\times}=K$, probably in the vicinity of the point $J_{\|}=J_{\perp}=K$ on the line $J_{\times}=0$. However, it is important to address the question of whether this crossover is a phase transition or a continuous change. As shown in Ref. 23 , the $k=2 \mathrm{SU}(2) \mathrm{WZW}$ model is unstable in the sense that it contains relevant operators which induce a flow to a stable $k=1 \mathrm{SU}(2) \mathrm{WZW}$, and one would therefore expect another second-order phase transition in the universality class of the $c=1 \mathrm{WZW}$ model. The alternatives to this scenario are a first-order phase transition or a continuous crossover; a definitive statement is not possible on the basis of the present considerations alone.

Because this special point or line cannot be expected to be accessible by RG analysis from either weak- or strongcoupling limits, it is natural to try to reach these points as a result of the flow away from the $c=3$ critical region defined in Sec. II B 3 [Eq. (18)]. We thus consider the effect of relevant and marginal perturbations on the corresponding CFT originating from the different interactions in the Hamiltonian (1).

\section{B. SU(4)-symmetric basis}

It is clear that the most convenient way to obtain the general continuum limit is by direct fermionization of the $X$ operators which are the generators of the su(4) algebra. Before this we perform a canonical transformation of the $X_{i}^{10}$ and $X_{i}^{30}$ operators for all lattice sites $i$,

$$
\begin{aligned}
& \widetilde{X}_{i}^{10}=\frac{1}{\sqrt{2}} i\left(X^{10}+i X^{30}\right) \rightarrow X_{i}^{10}, \\
& \widetilde{X}_{i}^{30}=\frac{1}{\sqrt{2}} i\left(X^{10}-i X^{30}\right) \rightarrow X_{i}^{30},
\end{aligned}
$$

from which one obtains [cf. Eqs. (6) and (7)]

$$
P_{i}^{\|}=\sum_{\alpha \beta} X_{i}^{\alpha \beta} X_{i+1}^{\beta \alpha}
$$

$$
\begin{gathered}
A_{i}^{\dagger}=\sum_{\alpha} X_{i}^{\alpha 0} X_{i+1}^{\alpha 0}, \quad B_{i}^{\dagger}=\sum_{a} X_{i}^{00} X_{i+1}^{00}-X_{i}^{a 0} X_{i+1}^{a 0}, \\
E_{i}^{+}=A_{i}^{\dagger} A_{i}, \quad E_{i}^{-}=B_{i}^{\dagger} B_{i},
\end{gathered}
$$

where $\alpha, \beta=0, \ldots, 3$, and $a=1,2,3$.

The generators of the su(4) algebra may be represented by four Dirac fermions,

$$
X_{k}^{\alpha \beta}=\frac{1}{2}\left(c_{k, \beta} c_{k, \alpha}^{\dagger}-c_{k, \alpha}^{\dagger} c_{k, \beta}\right)
$$

subject to the local constraint $\sum_{\alpha=0}^{3} c_{k, \alpha}^{\dagger} c_{k, \alpha}=1$. In terms of these variables the $Z_{4}$ transformation (13) is simply a particular case of the $\mathrm{U}(1)$ canonical transformation for $c_{k, 0}$ fermions,

$$
c_{k, 0} \rightarrow i c_{k, 0}, \quad c_{k, 0}^{\dagger} \rightarrow-i c_{k, 0}^{\dagger} .
$$

The fermionized version of the Hamiltonian (5) then takes the form

$$
\begin{aligned}
H= & \sum_{i, a, b} g_{1}\left(c_{i, a}^{\dagger} c_{i, b} c_{i+1, b}^{\dagger} c_{i+1, a}-\gamma c_{i, a}^{\dagger} c_{i, b} c_{i+1, a}^{\dagger} c_{i+1, b}\right) \\
& +g_{2}\left(c_{i, a}^{\dagger} c_{i, 0} c_{i+1,0}^{\dagger} c_{i+1, a}+c_{i, 0}^{\dagger} c_{i, a} c_{i+1, a}^{\dagger} c_{i+1,0}\right) \\
& +g_{3}\left(c_{i, a}^{\dagger} c_{i, 0} c_{i+1, a}^{\dagger} c_{i+1,0}+c_{i, 0}^{\dagger} c_{i, a} c_{i+1,0}^{\dagger} c_{i+1, a}\right) \\
& +g_{4}\left(c_{i, a}^{\dagger} c_{i, a}+c_{i+1, a}^{\dagger} c_{i+1, a}\right)+g_{5}\left(1-c_{i, a}^{\dagger} c_{i, a}\right) \\
& \times\left(1-c_{i+1, a}^{\dagger} c_{i+1, a}\right)
\end{aligned}
$$

where $g_{1}=\frac{1}{2}\left(J_{\|}+J_{\times}+K\right), \quad g_{2}=\frac{1}{2}\left(J_{\|}-J_{\times}+K\right), \quad g_{3}=\frac{1}{2}\left(J_{\|}\right.$ $\left.-J_{\times}-K\right), g_{4}=\left(J_{\perp}+2 K\right), g_{5}=2 K$, and $\gamma=1$. Note that we have retained the variable $\gamma$ as an additional perturbative parameter. 
In the low-energy limit the lattice fermions are expressed in terms of left- and right-moving fermions, $\psi_{L}$ and $\psi_{R}$ at the Fermi points,

$$
\frac{1}{\sqrt{a}} c_{n, \alpha} \simeq \psi_{L, \alpha} \exp \left(-i k_{F} x\right)+\psi_{R, \alpha} \exp \left(i k_{F} x\right),
$$

where $x=n a, \pm k_{F}= \pm \pi / r a, a$ is the lattice constant, and $r$ the filling factor, which is related to the group dimension [2 for $\mathrm{SU}(2), 4$ for $\mathrm{SU}(4)$ ]. In the continuum limit the different operators may be classified according to their scaling dimensions in the vicinity of the conformally invariant points.

Perturbation around CFT with $c=3$. For the particular set of values of the interaction parameters $J_{\|} / K=1, J_{\times} / K$ $=0$, which corresponds to a point in the $Z_{4}$-symmetric plane, the Hamiltonian may be written as [Eqs. (35) and (5)]

$$
\begin{aligned}
H= & K \sum_{i}\left[P_{i}^{\|}-\frac{1}{2}\left(E_{i}^{+}+E_{i}^{-}\right)+2 X_{i}^{00} X_{i+1}^{00}\right] \\
& -\left(J_{\perp}+2 K\right) \sum_{i} X_{i}^{00} .
\end{aligned}
$$

From the fermionic representation (36) one observes that Eq. (40) corresponds to the Hamiltonian (38) with the specific coupling values $g_{1}=g_{2}=g_{5} / 2=K, g_{3}=0, \quad \gamma=1$, and $g_{4}$ $=J_{\perp}+2 K$.

In Sec. II B 3 we have discussed the soluble model which has $c=3$ critical behavior in the region with finite rung and rung-rung interactions. Because this model is equivalent to the Hamiltonian (40) when the second term vanishes identically $(\gamma=0)$, it would appear natural that the critical region be extended by an increase of the positive rung-rung interaction. We therefore consider the perturbation of this critical model caused by the term $\left(E_{i}^{+}+E_{i}^{-}\right)$. In the continuum limit it is clear that the $\gamma$ term breaks the $\mathrm{SU}(4)$ symmetry,

$$
c_{i, a}^{\dagger} c_{i, b} c_{i+1, a}^{\dagger} c_{i+1, b} \simeq \psi_{L, a}^{\dagger} \psi_{L, b} \psi_{R, a}^{\dagger} \psi_{R, b}+\cdots,
$$

producing a marginally relevant product of currents of the SU(4)-symmetric WZW model for $\gamma>0$. The RG analysis ${ }^{47}$ shows directly that this interaction opens an exponential gap and drives the system into a dimerized state. A similar effect occurs in the spin-orbital model with symmetry-breaking perturbations. ${ }^{36,37}$ We note also that in the study of Ref. 48 this term appears as the continuum limit of the generators of the Temperley-Lieb algebra. ${ }^{26}$ In the present analysis the combination $\frac{1}{2}\left(E_{i}^{+}+E_{i}^{-}\right)-X_{i}^{00} X_{i+1}^{00}$ is the generator of the Temperley-Lieb algebra in the projected three-state-per-site subspace (further details are provided in Ref. 22).

We have shown that the SU(4)-symmetric model (38) has an exponential gap, and this is expected to persist up to $\gamma$ $\approx 1$, at which point the model becomes that of Eq. (1) with parameters $J_{\|} / K=1, J_{\times} / K=0$. Thus in the ring-exchange model (1) not only this point is gapped but also the region around it in the $J_{\perp}$ direction. We note that the rung interaction $\left[g_{4}\right.$ in Eq. (38)] induces both relevant (scaling dimension $3 / 2$ ) and marginal perturbations away from criticality.

One may develop a perturbative analysis for the entire problem by starting from this SU(4)-symmetric solution. The

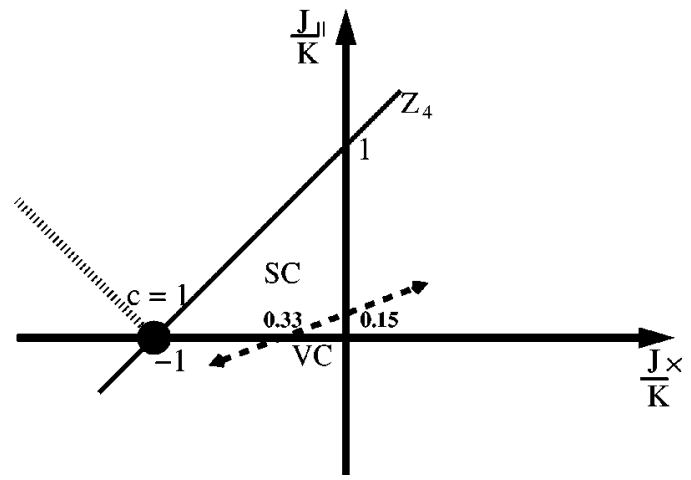

FIG. 10. Cross section of the phase diagram (Fig. 2) in the plane $J_{\perp}=0$. The solid line marks the projection of the plane of $Z_{4}$ symmetry, and the solid circle the line $\mathrm{AB}$ of massless, incommensurate solutions. The dashed line represents the second BabujianTakhtajan transition in the strong-coupling regime, which separates the scalar- and vector-chirality phases. The half plane in which a massless region exists around the point $J_{\times}=-K$ is represented by the dotted line.

$\mathrm{SU}(4)_{k=1}$ CFT allows a decomposition into the direct sum of two SU(2) $)_{k=2}$ CFTs, a fact used in the analysis of the SU(4)-symmetric spin-orbital model in Ref. 37. The addition of different perturbations to the SU(4)-symmetric solutions then leads to a lowering of the symmetry, and may induce a flow to one of the $\mathrm{SU}(2)_{k=2}$ components. These correspond to the two second-order phase transitions with $c=3 / 2$ at weak and strong coupling. The spin density is expressed in terms of the SU(4) primary fields and therefore, in addition to the uniform $(k=0)$ and staggered $(k=\pi)$ parts, has an oscillating part with momentum $k=\pi / 2$. This result appears plausible for the problem at hand in that a semiclassical analysis $^{11}$ reveals the presence of a ground state with orthogonal spin alignment on neighboring sites. We will not dwell further on this approach here.

\section{C. $c=1$ CFT}

We conclude this section by considering perturbations around the $c=1$ CFT solution given by Eq. (15). Although the point $J_{\perp}=0$ and line $\mathrm{AB}$ on the phase diagram of Fig. 2 possess additional degeneracies, the conformal dimensions of the model are unaltered. The WZW model with $c=1$ contains one primary matrix field of dimension $\left(\frac{1}{4}, \frac{1}{4}\right)$, and the perturbation caused by this field is related to dimerization or alternation, i.e., it breaks explicitly the symmetry of translation by one lattice site. This type of interaction is absent in the initial Hamiltonian (1), and therefore the relevant perturbations due to this matrix field are disallowed by symmetry. The only possible perturbation is then a marginal currentcurrent interaction which is present in the $g_{3}$ term of Eq. (38). Perturbation around the exact solution given by Eq. (15) requires that all other couplings be set to zero. This corresponds to the ray $J_{\|}+J_{\times}+K=0$ and $J_{\perp}=0$ in the parameter space of the model, i.e., to the fermionized Hamiltonian 


$$
\begin{aligned}
H= & \frac{1}{2} \sum_{i, a}\left[\left(c_{i, a}^{\dagger} c_{i, 0} c_{i+1,0}^{\dagger} c_{i+1, a}+c_{i, 0}^{\dagger} c_{i, a} c_{i+1, a}^{\dagger} c_{i+1,0}\right)\right. \\
& \left.+\left(1-2 c_{i, a}^{\dagger} c_{i, a}\right)\left(1-2 c_{i+1, a}^{\dagger} c_{i+1, a}\right)\right] \\
& +g\left(c_{i, a}^{\dagger} c_{i, 0} c_{i+1, a}^{\dagger} c_{i+1,0}+c_{i, 0}^{\dagger} c_{i, a} c_{i+1,0}^{\dagger} c_{i+1, a}\right),
\end{aligned}
$$

where $g=J_{\|}-J_{\times}-K$, and with the condition $J_{\|}+J_{\times}+K$ $=0$, which follows from Eq. (38). The general analysis of Ref. 47 is now applicable: when $g \equiv g_{3}<0$ the symmetrylowering term is marginally relevant and generates a gap, while if $g_{3}>0$ the term is marginally irrelevant and one expects a massless region to extend along this ray, as represented in Fig. 10. The point $g_{3}=0$ represents a type of Berezinsky-Kosterlitz-Thouless transition, as shown already from the exact solution in Sec. II. From the ray equation $J_{\|}+J_{\times}+K=0$, the coupling $g$ is proportional to $J_{\|}$, and thus for $J_{\|}>0$ one expects massless behavior, while for $J_{\|}$ $<0$ the dynamical generation of a Haldane gap occurs. One may further expect that the massless behavior persists for a small region of values of $J_{\perp}$ around this ray in the plane $J_{\|}+J_{\times}+K=0$. For values of the parameters such that the phases develop a gap, incommensurate behavior is expected, in the form of a maximum in the structure function $S(q)$ at values $q=q_{\max }$ where $0<q_{\max }<\pi$, over a broad region in the vicinity of the line $\mathrm{AB}$ in Fig. 2.

\section{Universality classes}

The preceding sections have revealed the rich critical behavior of the system under investigation. There exist two critical surfaces described by CFTs with $c=3 / 2$, the weakand strong-coupling regimes, and one critical region in the universality class of a $c=1 \mathrm{CFT}$. The existence of further second-order phase transitions may be excluded on the basis of general arguments. Both the weak-coupling and the corresponding strong-coupling regions of the phase diagram may be obtained by considering perturbations around the fixed points of two decoupled spin chains. This corresponds to the flow from $c=2$ CFTs to $c=3 / 2$ CFTs: the $c=3 / 2$ theories represent unstable fixed points, and a small change of the couplings in the Hamiltonian leads to further flow to the stable $c=1$ CFT. For the general model (1) with SU(2) symmetry, the latter CFT has the lowest possible central charge. These considerations are consistent with the Zamolodchikov theorem. As discussed in Sec. IV B, perturbations around the SU(4)-symmetric point reduce the symmetry and open a gap, thus excluding the possibility of $c$ $=3$ criticality. A candidate for this symmetry breaking is $\mathrm{SU}(4) \rightarrow \mathrm{SU}(3)$; however, the exactly soluble critical model with $c=2$, which has SU(3) symmetry, ${ }^{22}$ is "too far" from the parameter space of the current model, in the sense that it has a positive diagonal-diagonal coupling $V_{D D}$, and addition of interaction terms to this model induces a flow to a massive phase. We therefore conclude that the list of second-order phase transitions presented in this section is complete.

\section{SUMMARY AND DISCUSSION}

We now collect all of the information presented in the preceding sections. The phase diagram is shown in Fig. 2 in
3D form with coordinate axes $J_{\|} / K, J_{\times} / K$, and $J_{\perp} / K$. We have chosen this convention to highlight the phases and phase transitions obtained at strong $K$, as relatively little is known about this regime. The parallel dot-dashed lines demarcate the $Z_{4}$-symmetric plane, which intersects the horizontal plane on the line $J_{\times} / K=-1$. This line lies parallel to the coordinate axis $J_{\perp} / K$, and the part $-4<J_{\perp} / K<4$, represented by the thick dashed line between points $\mathrm{A}$ and $\mathrm{B}$, forms a critical incommensurate region emerging from the exact solution obtained in Sec. II B and discussed in Sec. III.

The matrix-product ansatz (Sec. III) reveals two lines of phase transitions, marked by the straight, solid lines in the $Z_{4}$-symmetric plane at $J_{\perp} / K= \pm 4$. The line $\mathrm{AA}^{\prime}$ at $J_{\perp} / K$ $=4$ represents a continuum of second-order phase transitions from a rung-singlet phase (Fig. 4) to a staggered dimer phase (Fig. 6), the existence of which has been confirmed by a number of studies. ${ }^{13,15,16}$ In Fig. 2 we have shown only this line, which is known exactly, although in fact it constitutes a part of a surface. The line $\mathrm{BB}^{\prime}$ at $J_{\perp} / K=-4$ denotes a continuum of first-order phase transitions from a ferromagnetic phase (Fig. 5) to a form of AKLT state (Fig. 9). Both lines intersect the incommensurate line at their ends, which thus represent two multicritical points (A and B). We have deduced (Sec. IV) the presence of another line (surface) of second-order phase transitions, the presence of which is consistent with the $Z_{4}$ symmetry of the plane in which it is in principle known exactly, and the conjectured form of the surface is represented both by the shaded triangle in the region close to the origin of Fig. 2 and by the dashed line in Fig. 10. This phase transition separates the strong-coupling vector-chirality phase (Fig. 8), dual to the rung-singlet phase, from the scalar-chirality phase (Fig. 7), which is dual to the staggered dimer phase. While the letters indicating the locations of the phases in Fig. 2 refer in a strict sense only to the plane of exact $Z_{4}$ symmetry, the absence of phase transitions other than those present on the diagram means that the gapped phases obtained for model parameters outside the plane are connected continuously to the exactly known states, and their physical properties evolve continuously as the values of the interactions are further changed away from the $Z_{4}$ plane.

Renormalization-group techniques and perturbative approaches applied around the exactly known solutions may be used to determine the fixed-point structure of the entire phase diagram. The $Z_{4}$-symmetric plane is found also to constitute the transition region between weak- and strong-coupling regimes, which is centered on the star in the phase diagram of Fig. 2. Analysis of the relevance of the additional terms in the model perturbing the $c=3$ CFT (Sec. IV) suggest that all points on this plane, other than the transition lines of the preceding paragraph and the incommensurate line $\mathrm{AB}$, have gapped excitations. These spin gaps are generated only by marginal symmetry-breaking perturbations and are therefore small, a result which was confused with gapless behavior in the numerical studies of Ref. 14. Within the plane, the RG fixed points corresponding to the $c=3 / 2$ transitions are unstable (Sec. V), and therefore we expect a flow to a stable $c=1$ theory, for which the obvious candidate is the incommensurate line bounded by the multicritical points $\mathrm{A}$ and $\mathrm{B}$. 
There is a certain mathematical and physical similarity between the behavior of the $S=1 / 2$ ladder with a ringexchange interaction and the $S=1$ bilinear-biquadratic model described by the Hamiltonian

$$
H_{B B}=\sum_{i}\left(\mathbf{S}_{i} \cdot \mathbf{S}_{i+1}\right)+\alpha\left(\mathbf{S}_{i} \cdot \mathbf{S}_{i+1}\right)^{2} .
$$

For different values of the parameter $\alpha$ this model contains a variety of phases and different forms of critical behavior. For $\alpha=-1$, the Babujian-Takhtajan model, ${ }^{46}$ it is in the same universality class as the two $c=3 / 2$ second-order phasetransition lines of the ring-exchange model. The Hamiltonian (43) also shows incommensurate behavior for the parameter region $1 / 3<\alpha<1$. However, in the bilinear-biquadratic model this is restricted to a gapped phase, in contrast to the current model where the critical incommensurate regime is related to the degeneracy of the $c=1 \mathrm{CFT}$. We comment that an explicit mapping from the $S=1$ model of Eq. (43) to a $S=1 / 2$ ladder system may be obtained by using a compositespin representation, ${ }^{18}$ but that the resulting model does not lie in the phase space (Fig. 2) of a ladder with ring-exchange interactions (see Sec. I).

The most accurate and extensive numerical studies performed to date for a ladder system with ring exchange are contained in Ref. 16. The first quadrant of the circular phase diagram presented in this work corresponds to the line $\left(J_{\|}\right.$ $\left.=J_{\perp}>0, J_{\times}=0\right)$ in Fig. 2 . We note the complete agreement of our analysis with the numerical investigation for this line: the four phases of Ref. 16 are those represented schematically in Figs. 4, 6, 7, and 8, and related under the $Z_{4}$ transformation as shown in Fig. 3. The complete phase diagram allows us not only to verify the Babujian-Takhtajan nature of the rung singlet to staggered dimer phase transition but to confirm that the scalar-chirality to vector-chirality phase transition lies in the same universality class, and in addition to specify the location $\left(J_{\|}=K\right)$ and nature (Sec. IV) of the crossover between staggered dimer and scalar-chirality phases, which remained unclear from the numerical analysis. The second quadrant of Fig. 1 of Ref. 16 is represented by the line $\left(J_{\|}=J_{\perp}<0, J_{\times}=0\right)$ in Fig. 2. Although we have not obtained specific information concerning this region of the phase diagram, the properties of the regions to which it is connected continuously allow us to deduce that the vectorchirality phase should be separated from the ferromagnetic phase by first-order transitions to a form of AKLT state, which indeed exhibits the collinear-spin property found by DMRG. Because our considerations do not include negative values of $K$, we refrain from comment on the third and fourth quadrants of the circular phase diagram.

Finally, we summarize briefly the relevance of our results for the materials and higher-dimensional systems mentioned in Sec. I. Experimentally determined values of $K$ for cuprate systems, including the ladder compound $\mathrm{La}_{6} \mathrm{Ca}_{8} \mathrm{Cu}_{24} \mathrm{O}_{41}$, suggest that the ring-exchange interaction may in fact be close to the value required to drive the rung-singlet phase to a staggered dimer state, and thus that staggered dimer correlations may be detectable. In two dimensions one expects that a larger value of $K$ would be required to find analogs of the more exotic ( i.e., chiral) phases of the model, and thus that these would most likely be detectable, if at all, as excitations. Films of ${ }^{3} \mathrm{He}$ have been found to offer larger values of $K$, but these appear both in combination with other multiple-spin-exchange processes and on a more complex lattice geometry which may lead to further topological possibilities for spin configurations. Our results suggest that this restricted-geometry system may provide a very rich spectrum of possible phases, but a considerably more specific analysis would be required.

In conclusion, we have analyzed a general model for a $S=1 / 2$ ladder with ring-exchange interactions. By investigating the exactly soluble points within the parameter space we obtain a complete classification of the phases and phase transitions in this type of system. Although we have considered the minimal model possessing both cyclic four-spin interactions and nontrivial exact solutions, we find a rich variety of gapped and gapless phases, of first- and second-order transitions, and of commensurate and incommensurate excitations, all connected by a complex renormalization-group flow pattern. The full phase diagram provides significant additional insight into the types of phases and transitions arising in low-dimensional spin systems as a consequence of the cooperation and competition between nearest-neighbor antiferromagnetic exchange interactions and multiple-spin interactions of the ring-exchange type.

\section{ACKNOWLEDGMENTS}

We would like to thank F. C. Alcaraz for explanations concerning the model of Eq. (15). We are grateful to A. Ferraz, P. Horsch, G. I. Japaridze, A. Läuchli, and C. Lhuillier for helpful communications and discussions. This work was supported by the Swiss National Science Foundation through Grant No. 20-68047.02.

\section{APPENDIX}

We summarize here the important formulas used in Sec. IV, and describe the fermionization procedure for the general bilinear-biquadratic spin-1/2 ladder in the limits of two decoupled chains.

The continuum limit of the SU(2) Heisenberg model is described by an SU(2) WZW model at level $k=1$. This model has one matrix primary operator $g_{\alpha}(\alpha=0,1,2,3,4)$ of scaling dimension $(1 / 4,1 / 4)$. The right and left Kac-Moody currents are fields of dimension $(1,0)$ and $(0,1)$. The relationship between these operators and the spin-operator density is given in the continuum limit by Eq. (25).

A pair of level-1 SU(2) WZW models may be represented in terms of four Ising fields. The operator content of these Ising models provides a set of elementary variables for constructing the continuum limit of the general ladder model. The critical Ising model is described by a $c=1 / 2$ CFT in the continuum limit. It contains holomorphic and antiholomorphic fields, respectively, $\psi(z)$ and $\bar{\psi}(\bar{z})$, with conformal dimensions $\left(\frac{1}{2}, 0\right)$ and $\left(0, \frac{1}{2}\right)$. The energy operator $\epsilon(z, \bar{z})$ $=i \psi(z) \bar{\psi}(\bar{z})$ has dimension $\left(\frac{1}{2}, \frac{1}{2}\right)$, while the order field 
$\sigma(z, \bar{z})$ and disorder field $\mu(z, \bar{z})$, related to the order field by Kramers-Wannier duality, have the same dimension $\left(\frac{1}{16}, \frac{1}{16}\right)$. The holomorphic and antiholomorphic components of energy-momentum tensor are $T(z)=-\frac{1}{2} \psi \partial \psi$ and $\bar{T}(\bar{z})=$ $-\frac{1}{2} \bar{\psi} \bar{\partial} \bar{\psi}$.

The representation of the fundamental level-1 SU(2) $\times \mathrm{SU}(2)$ WZW fields is given in terms of four Ising models by (see, e.g., Ref. 44)

$$
\begin{aligned}
& g_{0}=\sigma_{1} \sigma_{2} \sigma_{3} \sigma_{0}+\mu_{1} \mu_{2} \mu_{3} \mu_{0}, \\
& g_{1}=\mu_{1} \sigma_{2} \sigma_{3} \mu_{0}-\sigma_{1} \mu_{2} \mu_{3} \sigma_{0}, \\
& g_{2}=\sigma_{1} \mu_{2} \sigma_{3} \mu_{0}+\mu_{1} \sigma_{2} \mu_{3} \sigma_{0}, \\
& g_{3}=\sigma_{1} \sigma_{2} \mu_{3} \mu_{0}-\mu_{1} \mu_{2} \sigma_{3} \sigma_{0}, \\
& g_{0}^{\prime}=\sigma_{1} \sigma_{2} \sigma_{3} \sigma_{0}-\mu_{1} \mu_{2} \mu_{3} \mu_{0}, \\
& g_{1}^{\prime}=-\mu_{1} \sigma_{2} \sigma_{3} \mu_{0}-\sigma_{1} \mu_{2} \mu_{3} \sigma_{0}, \\
& g_{2}^{\prime}=-\sigma_{1} \mu_{2} \sigma_{3} \mu_{0}+\mu_{1} \sigma_{2} \mu_{3} \sigma_{0}, \\
& g_{3}^{\prime}=-\sigma_{1} \sigma_{2} \mu_{3} \mu_{0}-\mu_{1} \mu_{2} \sigma_{3} \sigma_{0},
\end{aligned}
$$

and the expressions for the $\mathrm{SU}(2) \times \mathrm{SU}(2)$ Kac-Moody currents are

$$
\begin{gathered}
J_{1}=\frac{1}{2} i\left(\psi_{1} \psi_{0}-\psi_{2} \psi_{3}\right), \\
J_{2}=\frac{1}{2} i\left(\psi_{2} \psi_{0}-\psi_{3} \psi_{1}\right), \\
J_{3}=\frac{1}{2} i\left(\psi_{3} \psi_{0}-\psi_{1} \psi_{2}\right), \\
J_{1}^{\prime}=-\frac{1}{2} i\left(\psi_{1} \psi_{0}+\psi_{2} \psi_{3}\right), \\
J_{2}^{\prime}=-\frac{1}{2} i\left(\psi_{2} \psi_{0}+\psi_{3} \psi_{1}\right), \\
J_{3}^{\prime}=-\frac{1}{2} i\left(\psi_{3} \psi_{0}+\psi_{1} \psi_{2}\right) .
\end{gathered}
$$

The fields $g_{i}(i=1,2,3)$ represent the staggered part of the spin-density operator, while the Kac-Moody currents correspond to its uniform part.

The operator-product expansions between Ising-model fields are ${ }^{45}$

$$
\begin{gathered}
\sigma(z, \bar{z}) \sigma(w, \bar{w}) \sim \frac{1}{|z-w|^{1 / 4}}+\frac{1}{2}|z-w|^{3 / 4} \epsilon(w, \bar{w}), \\
\mu(z, \bar{z}) \mu(w, \bar{w}) \sim \frac{1}{|z-w|^{1 / 4}}-\frac{1}{2}|z-w|^{3 / 4} \epsilon(w, \bar{w}), \\
\sigma(z, \bar{z}) \mu(w, \bar{w}) \sim \frac{\gamma(z-w)^{1 / 2} \psi(w)+\gamma^{*}(\bar{z}-\bar{w})^{1 / 2} \bar{\psi}(\bar{w})}{\sqrt{2}|z-w|^{1 / 4}}, \\
\mu(z, \bar{z}) \sigma(w, \bar{w}) \sim \frac{\gamma^{*}(z-w)^{1 / 2} \psi(w)+\gamma(\bar{z}-\bar{w})^{1 / 2} \bar{\psi}(\bar{w})}{\sqrt{2}|z-w|^{1 / 4}},
\end{gathered}
$$

and

$$
\begin{gathered}
\psi(z) \psi(w) \sim \frac{1}{z-w}+2(z-w) T(w), \\
\bar{\psi}(z) \bar{\psi}(w) \sim \frac{1}{\bar{z}-\bar{w}}+2(\bar{z}-\bar{w}) \bar{T}(\bar{w}), \\
\psi(z) \sigma(w, \bar{w}) \sim \frac{\gamma \mu(w, \bar{w})}{\sqrt{2}(z-w)^{1 / 2}}, \\
\psi(z) \mu(w, \bar{w}) \sim \frac{\gamma^{*} \sigma(w, \bar{w})}{\sqrt{2}(z-w)^{1 / 2}}, \\
\bar{\psi}(\bar{z}) \sigma(w, \bar{w}) \sim \frac{\gamma^{*} \mu(w, \bar{w})}{\sqrt{2}(\bar{z}-\bar{w})^{1 / 2}}, \\
\bar{\psi}(\bar{z}) \mu(w, \bar{w}) \sim \frac{\gamma \sigma(w, \bar{w})}{\sqrt{2}(\bar{z}-\bar{w})^{1 / 2}}, \\
\epsilon(z, \bar{z}) \epsilon(w, \bar{w}) \sim \frac{1}{|z-w|^{2}},
\end{gathered}
$$

where $\gamma=\exp (i \pi / 4)$.

These relations allow one to compute the OPEs between marginal operators defined in Eq. (27),

$$
\begin{gathered}
O_{1}(z) O_{1}(w) \sim \frac{3}{|z-w|^{4}}-\frac{2}{|z-w|^{2}} O_{1}+\cdots, \\
O_{2}(z) O_{2}(w) \sim \frac{3}{|z-w|^{4}}-\frac{2}{|z-w|^{2}} O_{1}+\cdots, \\
O_{1}(z) O_{2}(w) \sim \frac{-2 O_{2}}{|z-w|^{2}}+\cdots,
\end{gathered}
$$

$$
\begin{aligned}
& \left(\psi_{1} \bar{\psi}_{1}\right)(z) O_{1}(w) \sim \frac{-\left(\psi_{2} \bar{\psi}_{2}\right)(w)-\left(\psi_{3} \bar{\psi}_{3}\right)(w)}{|z-w|^{2}}, \\
& \left(\psi_{2} \bar{\psi}_{2}\right)(z) O_{1}(w) \sim \frac{-\left(\psi_{1} \bar{\psi}_{1}\right)(w)-\left(\psi_{3} \bar{\psi}_{3}\right)(w)}{|z-w|^{2}},
\end{aligned}
$$$$
\left(\psi_{3} \bar{\psi}_{3}\right)(z) O_{1}(w) \sim \frac{-\left(\psi_{2} \bar{\psi}_{2}\right)(w)-\left(\psi_{1} \bar{\psi}_{1}\right)(w)}{|z-w|^{2}},
$$$$
\left(\psi_{a} \bar{\psi}_{a}\right)(z) O_{2}(w) \sim \frac{-\left(\psi_{0} \bar{\psi}_{0}\right)(w)}{|z-w|^{2}} \quad(a=1,2,3),
$$ 


$$
\begin{aligned}
& \left(\psi_{0} \bar{\psi}_{0}\right)(z) O_{2}(w) \\
& \quad \sim \frac{-\left(\psi_{1} \bar{\psi}_{1}\right)(w)-\left(\psi_{2} \bar{\psi}_{2}\right)(w)-\left(\psi_{3} \bar{\psi}_{3}\right)(w)}{|z-w|^{2}} .
\end{aligned}
$$

Because the scaling dimensions of all operators are known around CFT points, one may proceed to the continuum limit for the spin Hamiltonian around the limit of two decoupled chains described by the WZW model with $c=2$. There exist two variants of this limit, at weak and strong $K$, and it is convenient to study the continuum limit of the general Hamiltonian

$$
\begin{aligned}
H= & \sum_{i} J_{L}\left(\mathbf{S}_{i} \cdot \mathbf{S}_{i+1}+\mathbf{T}_{i} \cdot \mathbf{T}_{i+1}\right)+J_{D}\left(\mathbf{S}_{i} \cdot \mathbf{T}_{i+1}+\mathbf{T}_{i} \cdot \mathbf{S}_{i+1}\right) \\
& +J_{R} \mathbf{S}_{i} \cdot \mathbf{T}_{i}+V_{L L}\left(\mathbf{S}_{i} \cdot \mathbf{S}_{i+1}\right)\left(\mathbf{T}_{i} \cdot \mathbf{T}_{i+1}\right)+V_{R R}\left(\mathbf{S}_{i} \cdot \mathbf{T}_{i}\right) \\
& \times\left(\mathbf{S}_{i+1} \cdot \mathbf{T}_{i+1}\right)+V_{D D}\left(\mathbf{S}_{i} \cdot \mathbf{T}_{i+1}\right)\left(\mathbf{S}_{i+1} \cdot \mathbf{T}_{i}\right),
\end{aligned}
$$

where in addition to the leg $\left(J_{L}\right)$, rung $\left(J_{R}\right)$, and diagonal $\left(J_{D}\right)$ Heisenberg interactions we include leg-leg $\left(V_{L L}\right)$, rung-rung $\left(V_{R R}\right)$, and diagonal-diagonal $\left(V_{D D}\right)$ four-spin interactions. The most relevant contribution from the biquadratic terms arises from the product of the staggered parts of the corresponding composite quadratic expressions, and from the operator product expansion between quadratic product of currents with the quadratic products of the staggered parts,

$$
\begin{gathered}
\mathbf{S}_{i} \cdot \mathbf{S}_{i+1} \simeq\left(O_{1}+O_{2}\right)+(-1)^{x / a} \lambda g_{0}, \\
\mathbf{T}_{i} \cdot \mathbf{T}_{i+1} \simeq\left(O_{1}+O_{2}\right)+(-1)^{x / a} \lambda g_{0}^{\prime}, \\
\mathbf{S}_{i} \cdot \mathbf{T}_{i} \simeq \frac{1}{2}\left(O_{1}-O_{2}\right)-\left(\epsilon_{1}+\epsilon_{2}+\epsilon_{3}-3 \epsilon_{0}\right) \\
+(-1)^{x / a}:\left[g^{a}\left(J^{a^{\prime}}+\bar{J}^{a^{\prime}}\right)+\left(J^{a}+\bar{J}^{a}\right) g^{\prime a}\right]:, \\
\mathbf{S}_{i} \cdot \mathbf{T}_{i+1} \simeq \frac{1}{2}\left(O_{1}-O_{2}\right)+\left(\epsilon_{1}+\epsilon_{2}+\epsilon_{3}-3 \epsilon_{0}\right) \\
+(-1)^{x / a}:\left[g^{a}\left(J^{a^{\prime}}+\bar{J}^{a^{\prime}}\right)-\left(J^{a}+\bar{J}^{a}\right) g^{\prime a}\right]:,
\end{gathered}
$$

and

$$
\begin{aligned}
:\left(\mathbf{S}_{i} \cdot \mathbf{S}_{i+1}\right)::\left(\mathbf{T}_{i} \cdot \mathbf{T}_{i+1}\right): \simeq \lambda^{2}\left[-4\left(O_{1}+O_{2}\right)+6 \sum_{k=0}^{3} \epsilon_{k}\right], \\
:\left(\mathbf{S}_{i} \cdot \mathbf{T}_{i+1}\right)::\left(\mathbf{S}_{i+1} \cdot \mathbf{T}_{i}\right): \simeq \lambda^{2}\left[O_{1}-5 O_{2}+3 \epsilon_{0}\right. \\
\left.-5\left(\epsilon_{1}+\epsilon_{2}+\epsilon_{3}\right)+3 \sum_{k=0}^{3} \epsilon_{k}\right], \\
:\left(\mathbf{S}_{i} \cdot \mathbf{T}_{i}\right)::\left(\mathbf{S}_{i+1} \cdot \mathbf{T}_{i+1}\right): \simeq \\
\lambda^{2}\left[O_{1}-5 O_{2}-3 \epsilon_{0}\right. \\
\left.+5\left(\epsilon_{1}+\epsilon_{2}+\epsilon_{3}\right)-3 \sum_{k=0}^{3} \epsilon_{k}\right],
\end{aligned}
$$

where the cutoff-dependent constant $\lambda$ emerges from the operator product expansion between different contributions.

These expressions give in terms of Majorana fermions the continuum limit of the Hamiltonian, which is separated into singlet, triplet, and marginal parts as in Eq. (26), with

$$
\begin{gathered}
m_{t}=J_{R}-2 J_{D}-\lambda^{2}\left(6 V_{L L}-2 V_{D D}+2 V_{R R}\right), \\
m_{s}=-3 J_{R}+6 J_{D}-\lambda^{2}\left(6 V_{L L}+6 V_{D D}-6 V_{R R}\right), \\
\lambda_{1}=-4 J_{L}+J_{R} / 2+J_{D}+\left(-4 V_{L L}+V_{D D}+V_{R R}\right), \\
\lambda_{2}=-4 J_{L}-J_{R} / 2-J_{D}-\left(-4 V_{L L}-5 V_{D D}-5 V_{R R}\right),
\end{gathered}
$$

and operators $O_{1}$ and $O_{2}$ as defined in Eq. (27). This representation allows one to study simultaneously the limits of weak and strong $K$ (Sec. IV). We note that the signs of the four-spin interaction terms in the expression for the masses are different from those in Ref. 10 but the same as those resulting from the analysis in Ref. 21 of the string order parameter.
${ }^{1}$ R. Coldea, S.M. Hayden, G. Aeppli, T.G. Perring, C.D. Frost, T.E. Mason, S.-W. Cheong, and Z. Fisk, Phys. Rev. Lett. 86, 5377 (2001).

${ }^{2}$ M. Matsuda, K. Katsumata, R.S. Eccleston, S. Brehmer, and H.-J. Mikeska, Phys. Rev. B 62, 8903 (2000).

${ }^{3}$ M. Windt, M. Grüninger, T. Nunner, C. Knetter, K. Schmidt, G.S. Uhrig, T. Kopp, A. Freimuth, U. Ammerahl, B. Büchner, and A. Revcolevschi, Phys. Rev. Lett. 87, 127002 (2001); K.P. Schmidt, C. Knetter, and G.S. Uhrig, Europhys. Lett. 56, 877 (2001).

${ }^{4}$ M. Siqueira, J. Nyéki, B. Cowan, and J. Saunders, Phys. Rev. Lett. 78, 2600 (1997).

${ }^{5}$ E. Collin, S. Triqueneaux, R. Harakaly, M. Roger, C. Bäuerle, Yu.M. Bunkov, and H. Godfrin, Phys. Rev. Lett. 86, 2447 (2001)
${ }^{6}$ See G. Misguich, B. Bernu, C. Lhuillier, and C. Waldtmann, Phys. Rev. Lett. 81, 1098 (1998), and references therein; W. LiMing, G. Misguich, P. Sindzingre, and C. Lhuillier, Phys. Rev. B 62, 6372 (2000).

${ }^{7}$ M. Takahashi, S.M. Girvin, and D. Yoshioka, J. Phys. C 10, 1289 (1977); A.H. MacDonald, S.M. Girvin, and D. Yoshioka, Phys. Rev. B 37, 9753 (1988).

${ }^{8}$ E. Müller-Hartmann and A. Reischl, Eur. Phys. J. B 28, 173 (2002).

${ }^{9}$ S. Brehmer, H.-J. Mikeska, M. Müller, N. Nagaosa, and S. Uchida, Phys. Rev. B 60, 329 (1999).

${ }^{10}$ M. Müller, T. Vekua, and H.-J. Mikeska, Phys. Rev. B 66, 134423 (2002). 
${ }^{11}$ A. Chubukov, E. Gagliano, and C. Balseiro, Phys. Rev. B 45, 7889 (1988).

${ }^{12}$ A.A. Katanin and A.P. Kampf, Phys. Rev. B 66, 100403 (2002).

${ }^{13}$ K. Hijii and K. Nomura, Phys. Rev. B 65, 104413 (2002).

${ }^{14}$ Y. Honda and T. Horiguchi cond-mat/0106426 (unpublished).

${ }^{15}$ T. Hikihara, T. Momoi, and X. Hu, Phys. Rev. Lett. 90, 087204 (2003).

${ }^{16}$ A. Läuchli, G. Schmid, and M. Troyer, Phys. Rev. B 67, 100409(R) (2003).

${ }^{17}$ K. Hijii, S. Qin, and K. Nomura, Phys. Rev. B 68, 134403 (2003).

${ }^{18}$ Ö. Legeza, G. Fáth, and J. Sólyom, Phys. Rev. B 55, 291 (1997).

${ }^{19}$ M.P. M den Nijs and K. Rommelse, Phys. Rev. B 40, 4709 (1989).

${ }^{20}$ M. Nakamura and S. Todo, Phys. Rev. Lett. 89, 077204 (2002).

${ }^{21}$ G. Fáth, Ö. Legeza, and J. Sólyom, Phys. Rev. B 63, 134403 (2001).

${ }^{22}$ V. Gritsev and D. Baeriswyl, J. Phys. A: Math. Gen. 36, 12129 (2003).

${ }^{23}$ I. Affleck and F.D.M. Haldane, Phys. Rev. B 36, 5291 (1987).

${ }^{24}$ A.B. Zamolodchikov, Pis'ma Zh. Eksp. Teor. Fiz. 43, 565 (1986) [JETP Lett. 43, 730 (1986)].

${ }^{25}$ P.F. Arndt, T. Heinzl, and C.M. Yung, J. Phys. A 28, 323 (1995).

${ }^{26}$ The operators $P_{i, i+1}^{\|}, P_{i, i+1}^{\times}$and $E_{i, i+1}, \widetilde{E}_{i, i+1}$ are generators of the algebra

$$
\begin{gathered}
P_{i}^{2}=1, \quad P_{i} P_{i+1} P_{i}=P_{i+1} P_{i} P_{i+1}, \\
E_{i}^{2}=4 E_{i}, \quad E_{i} E_{i+1} E_{i}=E_{i}, \\
P_{i} E_{i}=E_{i} P_{i}=E_{i}, \\
P_{i} E_{i+1} E_{i}=P_{i+1} E_{i}, \quad E_{i+1} E_{i} P_{i+1}=E_{i+1} P_{i},
\end{gathered}
$$

for neighboring plaquettes, and

$$
P_{i} P_{j}=P_{j} P_{i}, \quad E_{i} E_{j}=E_{j} E_{i}, \quad P_{i} E_{j}=E_{j} P_{i}
$$

for $|i-j|>1$. We have used here the plaquette notation $P_{i, i+1}$ $\equiv P_{i}$ and $E_{i, i+1} \equiv E_{i}$, in which $P_{i}$ denotes either $P_{i}^{\|}$or $P_{i}^{\times}$and $E_{i}$ denotes either $E_{i}$ or $\widetilde{E}_{i}$. We note that there exist a number of other relations between the operators which follow from the above. The operators $P_{i}$ satisfy the braid-algebra relations, while the operators $E_{i}$ form a Temperley-Lieb algebra (Ref. 27). Together these operators form a Brauer (Ref. 28) [or braid monoid (Ref. 29)] algebra.
${ }^{27}$ H.N.V. Temperley and E.H. Lieb, Proc. R. Soc. London, Ser. A 322, 251 (1971).

${ }^{28}$ R. Brauer, Ann. Math. 38, 854 (1937).

${ }^{29}$ M. Wadati, T. Deguchi, and Y. Akutsu, Phys. Rep. 180, 247 (1989).

${ }^{30}$ L.D. Faddeev, Int. J. Mod. Phys. A 10, 1845 (1995).

${ }^{31}$ M.J. Martins and P.B. Ramos, Nucl. Phys. B 500, 579 (1997); Y. Cheng, M.L. Ge, and K. Xue, Commun. Math. Phys. 136, 195 (1991).

${ }^{32}$ S. Albeverio, S.M. Fei, and Y.P. Wang, Europhys. Lett. 47, 364 (1999); M.T. Batchelor and M. Maslen, J. Phys. A 33, 443 (2000).

${ }^{33}$ J.C. Bonner and M.E. Fisher, Phys. Rev. 135, A640 (1964).

${ }^{34}$ F.C. Alcaraz, D. Arnaudon, V. Rittenberg, and M. Scheunert, Int. J. Mod. Phys. A 9, 3473 (1994).

${ }^{35}$ K.I. Kugel and D.I. Khomskii, Zh. Eksp. Teor. Fiz. 64, 1429 (1973) [Sov. Phys. JETP 37, 725 (1973)].

${ }^{36}$ P. Azaria, A.O. Gogolin, P. Lecheminant, and A.A. Nersesyan, Phys. Rev. Lett. 83, 624 (1999).

${ }^{37}$ C. Itoi, S. Qin, and I. Affleck, Phys. Rev. B 61, 6747 (2000).

${ }^{38}$ Y. Wang, Phys. Rev. B 60, 9236 (1999).

${ }^{39}$ T. Momoi, T. Hikihara, M. Nakamura, and X. Hu, Phys. Rev. B 67, 174410 (2003).

${ }^{40}$ A.K. Kolezhuk and H.-J. Mikeska, Int. J. Mod. Phys. B 12, 2325 (1998).

${ }^{41}$ I. Affleck, T. Kennedy, E. Lieb, and H. Tasaki, Phys. Rev. Lett. 59, 799 (1987); Commun. Math. Phys. 115, 477 (1988).

${ }^{42}$ G. Müller, H. Thomas, H. Beck, and J.C. Bonner, Phys. Rev. B 24, 1429 (1981)

${ }^{43}$ A.A. Nersesyan and A.M. Tsvelik, Phys. Rev. Lett. 78, 3939 (1997); A. O. Gogolin, A. A. Nersesyan, and A. M. Tsvelik, Bosonization and Strongly Correlated Systems (Cambridge University Press, Cambridge, 1998).

${ }^{44}$ D. Allen and D. Sénéchal, Phys. Rev. B 55, 299 (1997).

${ }^{45}$ P. Di Francesco, P. Mathieu, and D. Sénéchal, Conformal Field Theory (Springer, New York, 1997).

${ }^{46}$ L.A. Takhtajan, Phys. Lett. A 87, 479 (1982); H.M. Babujian, ibid. 90, 479 (1982).

${ }^{47}$ C. Itoi and M.-H. Kato, Phys. Rev. B 55, 8295 (1997).

${ }^{48}$ I. Affleck, J. Phys.: Condens. Matter 2, 405 (1990). 